\title{
Regression-based Monte Carlo methods for stochastic control models: Variable annuities with lifelong guarantees
}

\author{
Yao Tung Huang and Yue Kuen Kwok* \\ Department of Mathematics \\ Hong Kong University of Science and Technology \\ Hong Kong, China
}

\begin{abstract}
We present the regression-based Monte Carlo simulation algorithms for solving the stochastic control models associated with pricing and hedging of the Guaranteed Lifelong Withdrawal Benefit (GLWB) in variable annuities, where the dynamics of the underlying fund value is assumed to evolve according to the stochastic volatility model. The GLWB offers a lifelong withdrawal benefit even when the policy account value becomes zero while the policyholder remains alive. Upon death, the remaining account value will be paid to the beneficiary as a death benefit. The bang-bang control strategy analyzed under the assumption of maximization of the policyholder's expected cash flow reduces the strategy space of optimal withdrawal policies to three choices: zero withdrawal, withdrawal at the contractual amount or complete surrender. The impact on the GLWB value under various withdrawal behaviors of the policyholder is examined. We also analyze the pricing properties of GLWB subject to different model parameter values and structural features.
\end{abstract}

JEL classification: G22, C50

Keywords: variable annuities, lifelong withdrawal guarantees, stochastic control models, Monte Carlo simulation, regression-based algorithms, stochastic volatility

\section{Introduction}

The market for variable annuities has shown phenomenal growth since 1990s. At the end of 2011, the total assets of variable annuities topped USD 1.5 trillion. As sweeteners to attract businesses, issuers of variable annuities provide various forms of guarantees riders to investors, besides equity participation with investment choices among a list of mutual funds. These guarantees can be classified into two broad types: guaranteed minimum death benefits (GMDBs) and guaranteed minimum living benefits (GMLBs). The second type can be further classified into three subtypes, namely, guaranteed minimum accumulation benefits (GMABs), guaranteed minimum income benefits (GMIBs) and guaranteed minimum withdrawal benefits (GMWBs). A good comprehensive summary of the structural features of these benefits can be found in Bauer et al. (2008) and Bacinello et al. (2011).

\footnotetext{
${ }^{*}$ Correspondence author, E-mail: maykwok@ust.hk. 24 July, 2014
} 
In this paper, we focus on the analysis of pricing and hedging issues of the latest variant of the GMWB, so called guaranteed lifelong withdrawal benefit (GLWB). This new form of guarantee combines withdrawal flexibility with longevity protection. In a typical variable annuity with GLWB, the policyholder pays a single lump sum payment to the issuer. The payment is then invested into the mutual funds of risky assets chosen by the policyholder. The benefit base is initially set to be the upfront payment. Under the lifelong withdrawal guarantee, the policyholder is entitled to withdraw a fixed proportion of the benefit base periodically (say, annual withdrawals) for life even when the policy fund account value has dropped to zero. Upon the death of the policyholder, the remaining amount in the policy fund account will be paid to the beneficiary. Another distinctive feature of GLWB is that the benefit base may grow over the life of the policy. Under the ratchet provision (step-up), the benefit base is increased to the policy fund account value on a ratchet date if the account value exceeds the previous benefit base recorded on the last withdrawal date. Under the bonus provision (roll-up), the benefit base may also be increased by a proportional amount if the policyholder chooses not to withdraw on the withdrawal date.

The pricing and hedging of guaranteed withdrawal benefits in variable annuities (GMWB and GLWB) pose a lot of technical challenges since the pricing model leads to an optimal stochastic control problem with complex path dependent features due to periodic withdrawals, ratchet and bonus features. The optimal withdrawal policies are not known a priori but they are solution to the stochastic controls in the pricing model. The academic research on GMWB starts with the pioneering works of Milevsky and Salisbury (2006), Dai et al.(2008) and Chen et al.(2008). These earlier pricing models of GMWB take the assumption of a geometric Brownian motion (GBM) for the underlying fund value process and continuous withdrawal process as the control in the derivation of the singular stochastic control models. More recently, Huang and Forsyth (2012) and Huang and Kwok (2014) manage to perform a complete characterization of the optimal withdrawal policies of the GMWB under the simplifying assumption of GBM and continuous withdrawal. The more recent research works on pricing GMWB show the departure from the simple GBM framework for the fund value processes to that under stochastic volatilities and / or stochastic interest rates. Peng et al. (2012) derive both the analytic upper and lower bounds for the fair values of GMWB under stochastic interest rates using analytic approximation methods. Donelly et al.(2014) consider numerical valuation of GMWB under both stochastic volatility and interest rates using the Alternating-Direction-Implicit method in their finite difference schemes. In the literature of pricing variable annuities under stochastic volatility, the Heston stochastic model (Heston, 1993) has been commonly used due to its nice analytic tractability. However, recent empirical studies show that the Heston form of affine square root stochastic volatility model is susceptible to misspecification of volatility dynamics (Christoffersen et al., 2010). Under the Heston model assumption of volatility dynamics, the instantaneous change in volatility should be Gaussian and homoscedastic. However, empirical studies on daily realized volatility changes are in conflict with the Gaussian and homoscedastic volatility implication of the Heston model. Christoffersen et al. (2010) argue that the best volatility specification is the ONE model with linear diffusion for variance instead of square root diffusion in the Heston model. By using the time series data on S\&P 500 daily return, Javaheri (2004) analyzes the CEV type instantaneous variance process and find that the 3/2-power in the exponent of the volatility of variance process performs the best. Ishida and Eagle (2002) estimate the power to be 1.71 for S\&P 500 daily return for a 30-year period. Indeed, nice analytical tractability in option valuation as exhibited by the Heston model has no distinctive advantage over the ONE model and 3/2-model when one uses the Monte Carlo simulation algorithms for numerical pricing of variable annuities. It is more relevant to adopt appropriate stochastic volatility models that provide better volatility 
specification.

The pricing models of GLWB can be considered as extensions of those of GMWB together with the inclusion of mortality risk. Shah and Bertsimas (2008) analyze the impact of various risk factors, like market risks and mortality risks, in the fair value of GLWB using the continuous time models. Under the assumption of static withdrawal strategy, Piscopo and Haberman (2011) analyze the impact of mortality risk using a flexible model of mortality dynamics on the fair value of GLWB. Holz et al. (2012) price the GLWB for different product design and model parameters under the GBM dynamics of the underlying fund value process. They also consider various forms of policyholder withdrawal behavior, including deterministic, probabilistic and stochastic models. They establish that the optimal strategy for a GLWB contract that does not include any step-up or roll-up feature consists only of either a withdrawal of the guaranteed amount or complete surrender. With this relatively simple set of withdrawal strategies, they show how to use the Monte Carlo simulation method to price the GLWB by following a similar dynamic programming procedure in the solution of optimal stopping problems. Steinorth and Mitchell (2012) adopt an expected utility framework to examine how a risk-averse decision maker would choose her optimal withdrawal policies. They show that the ratchet provision may make the policyholder behavior more predictable. Huang et al. (2013) consider the optimal initiation of a GLWB in a variable annuity, that is, the optimal time that the policyholder should end the accumulation phase and start withdrawals. They show that it is non-optimal to delay the withdrawal phase. This is intuitively obvious since the GLWB gains in value when the probability of "ruined" (zero policy fund value) is higher. At the time of "ruined", the proportional fee charges terminate. Therefore, it is indeed advantageous for the policyholder to observe the policy fund value to become zero. It also becomes worse to the issuer since the withdrawal guarantee kicks in. Under the simplified assumption of deterministic surrender rates, Kling et al. (2013) consider the model risks taken up by the insurer when constant equity volatilities are assumed for hedging purposes. They find that the fair values of the guarantees do not show significant change under either constant or stochastic volatility models. Using the Heston stochastic volatility model for the underlying fund value process, they show that the insurer's profit / loss and other risk measures may change dramatically due to the presence of vega risk. Also, these model risks can be reduced significantly if appropriate hedging strategies based on minimizing both the delta and vega risks are implemented. They also suggest how the issuer may reduce risk by better product design, say, limiting the inclusion of highly risky assets in the policyholder's fund account when market volatilities become high. Forsyth and Vetzal (2014) develop an implicit finite difference schemes for solving a coupled system of one-dimensional partial differential equations in the determination of the hedging cost for a GLWB variable annuity contract when the underlying fund value process follows a Markov regime switching process. They also consider the impact on the cost of hedging under various withdrawal policies, including the optimal withdrawal policies that maximize policyholder's expected value of cash flows and sub-optimal withdrawal policies that are dependent on moneyness of the surrender option.

The contribution of this paper is two-fold. First, we construct efficient regression-based Monte Carlo simulation algorithms for solving the stochastic control models associated with pricing of the GLWB in variable annuities under stochastic volatility. Efficiency of the simulation procedure is enhanced by the derivation of the closed form solution for the price function of the GLWB when the fund value becomes zero. By extending the bang-bang analysis of the set of control policies on withdrawal, we show that the strategy space of the optimal withdrawal policies consist of three choices only: zero withdrawal, withdrawal at the contractual amount or complete surrender. This paves the successful implementation of the regression-based approach for solving the optimal control and value function in the GLWB stochastic control model, using a similar dynamic programming 
procedure that is commonly adopted in the determination of the optimal stopping rule in an optimal stopping model (like an American option model). We also perform convergence analysis of the regression-based Monte Carol algorithms under the assumption of exact simulation of the simulated paths of the underlying fund value process. Second, we perform extensive studies on the pricing and hedging properties of the GLWB subject to varying values of the model parameters in the fund value process, like the volatility parameters, correlation coefficient between the fund value and stochastic volatility. Instead of choosing the Heston model for the dynamics of stochastic volatility, we adopt the ONE model and 3/2 model. These stochastic volatility models have been shown to have better specification of volatility dynamics compared to the Heston model. We also examine the impact of various structural features in the GLWB, like the penalty charge, bonus rate and ratchet feature, on the optimal withdrawal policies and complete surrender decision. Recall that the worst hedging cost of the GLWB is derived based on the assumption that the policyholder chooses the optimal withdrawal policies that maximize the discounted expectation of the future cash flows. However, various empirical studies have shown that policyholders may limit their choices in the withdrawal strategies, like forfeiting the choice of zero withdrawal or complete surrender or both. We consider the impact on the GLWB value under these suboptimal withdrawal behaviors of the policyholder.

This paper is organized as follows. In the next section, we present a product description of GLWB in a variable annuity and present the model formulation of GLWB under the assumption of the ONE and $3 / 2$ stochastic volatility models for the underlying fund value process. We discuss the complex features of the ratchet and bonus provisions, that determine the jump conditions on the value function, policy fund value and benefit base across withdrawal dates. In Section 3, we present the details of the regression-based Monte Carlo simulation algorithms for solving the stochastic control models of GLWB. The bang-bang analysis of the strategy space of optimal withdrawal policies indicates that there are only three possible optimal choices: zero withdrawal, withdrawal at the contractual amount and complete surrender. The dynamic programming procedure of comparing the surrender value and continuation value under either withdrawal of contractual amount or zero withdrawal can be applied. We also derive the closed form solution for the value function of GLWB when the policy fund value has dropped to zero. This helps save computational times on simulation runs since quite a significant portion of simulation paths lead to zero policy fund value before termination due to the occurrence of either the death event or complete surrender. Convergence proof of the regression-based algorithm for solving the stochastic control model of GLWB is presented. In Section 4, we analyze the pricing and hedging properties of GLWB subject to different market conditions, like equity volatilities and other model parameters in the stochastic volatility models. We examine the impact of different product design in the structural features on the optimal withdrawal policies. We also consider other withdrawal behaviors adopted by the policyholder and compare the profit and loss of the issuer under various withdrawal behaviors of the policyholder. The last section contains summary of results and conclusive remarks.

\section{Model formulation}

We start with a product description of the GLWB in a variable annuity contract. At time 0 , the policyholder pays an upfront single premium $P$ initially in her policy account, which is then invested in mutual funds of her own choice. We use $W_{t}$ to denote the policy fund value process, where $W_{0}=P$. The proportional fee charged by the issuer for the provision of the guarantee is taken from the policy account through the cancellation of fund units. The GLWB rider gives the policyholder the guaranteed withdrawals on preset withdrawal dates for 
life. The contractual withdrawal amount is calculated based on a fixed proportion of the benefit base $A_{t}$. The benefit base is set to be the upfront premium initially, where $A_{0}=P$. It can be adjusted upward via the ratchet provision (step-up) or bonus feature (roll-up). On a ratchet date, the ratchet mechanism increases the benefit base to the level of the policy account if the policy account value exceeds the benefit base on the previous withdrawal date. For the bonus feature, suppose the policyholder chooses not to withdraw any amount on a withdrawal date, then the benefit base is increased proportionally by the bonus rate. The policyholder is also allowed to withdraw more than the contractual withdrawal amount and the net amount received is subject to a proportional penalty charge. Complete surrender of the policy refers to the withdrawal of the whole policy account. As a result, the variable annuity contract terminates. Another event that causes the termination of the contract is the death of the policyholder. The value that remains in the policy account will be passed to a beneficiary. The issuer is faced with mortality risk, market risk and uncertainty in the surrender behavior. We assume that withdrawals are allowed on a predetermined set of dates. Upon the death of the policyholder, the remaining policy fund value is paid to the beneficiary on the next upcoming withdrawal date. Also, ratchet events may be allowed on all withdrawal dates or limited to a subset of withdrawal dates.

\section{Mortality risks and market risks}

The mortality risk is assumed to be diversifiable across a large number of policyholders. Let the inception date of the policy be time 0 . We follow the usual notation where the mortality tables are given in terms of integer ages. Let $T$ denote the maximum age beyond which survival is impossible. Let ${ }_{i} p_{x_{0}}$ denote the probability that an $x_{0}$-year old policyholder at time 0 still survives in the next $i$ years. Let $q_{x_{0}+i}$ denote the probability that an $x_{0}+i$ year old dies in the next year. With reference to a policyholder whose age is $x_{0}$, for simplicity of notation, we write ${ }_{i} p_{x_{0}}$ and $q_{x_{0}+i}$ as $p_{i}$ and $q_{i}$, respectively.

We extend the usual GBM assumption for the fund value process to allow for stochastic volatility. We let $W_{t}$ denote the policy fund value process and $\eta$ be the constant proportional fee charged on the policy fund that is paid by the policyholder for the provision of the guaranteed lifelong withdrawal benefit. For a probability space $(\Omega, \mathcal{F}, Q)$ with a filtration $\mathcal{F}=\left(\mathcal{F}_{t}\right)_{0 \leq t \leq T}$ satisfying the usual assumptions, we assume the existence of an equivalent (local) martingale measure $Q$ under which the process of the discounted spot price of an underlying asset is a (local) martingale. We consider the following general formulation of stochastic volatility models for the $Q$-dynamics of the underlying fund value process (Christoffersen et al., 2010), where the $Q$-dynamics of $W_{t}$ can be expressed as

$$
d W_{t}=(r-\eta) W_{t} d t+\sqrt{v_{t}} W_{t}\left[\rho d B_{t}^{(1)}+\sqrt{1-\rho^{2}} d B_{t}^{(2)}\right]
$$

and

$$
d v_{t}=\kappa v_{t}^{a}\left(\theta-v_{t}\right) d t+\epsilon v_{t}^{b} d B_{t}^{(1)}, \quad \text { for } a=\{0,1\} \text { and } b=\{1 / 2,1,3 / 2\} .
$$

Here, $B_{t}^{(1)}$ and $B_{t}^{(2)}$ are uncorrelated $Q$-Brownian motions, $\rho$ is the correlation coefficient, $\epsilon$ is the volatility of variance, $\kappa$ is the risk neutral speed of mean reversion, $\theta$ is the risk neutral long-term averaged variance, and $r$ is the riskless interest rate. To derive the above $Q$-dynamics of $v_{t}$, the following functional form for the variance risk premium: $\lambda(W, v, t)=\lambda_{0} v^{a+1}$ has been assumed, where $\lambda_{0}$ is a constant. The choice of $b=1 / 2$ (square root diffusion for variance) corresponds to the Heston model. Based on various empirical studies on volatility dynamics (Christoffersen et al., 2010; Ishida and Eagle, 2002; Javaheri, 2004), we adopt $b=1$ (ONE model $)$ and $b=3 / 2(3 / 2$ model $)$ while the Heston model is abandoned in our numerical calculations. In the above family of stochastic volatility models, it is common to choose $a=0$ and $b=1$ for the ONE model while 
$a=1$ and $b=3 / 2$ for the $3 / 2$-model.

Recall that $W_{0}=P$, where $P$ is the upfront payment at initiation of the policy contract. The contractual withdrawal amount is a fixed proportion $G$ of the benefit base. We let $A_{t}$ denote the value process of the benefit base, where $A_{0}=P$. Both $W_{t}$ and $A_{t}$ exhibit jumps across the withdrawal dates. The value function of the GLWB is denoted by $V(W, A, v, t)$, with dependence on the policy fund value $W$, benefit base $A$ and stochastic variance $v$.

\section{Jump conditions of the value function across a withdrawal date}

We assume annual withdrawal to take place on the set of discrete time points, $\mathcal{T}=\{1,2, \ldots, T-1\}$ in years. Let $\mathcal{T}_{e}$ be the set of discrete time points that correspond to the preset ratchet event dates, where $\mathcal{T}_{e} \subseteq \mathcal{T}$. The annual withdrawal amounts as characterized by $\Gamma=\left(\gamma_{1}, \gamma_{2}, \ldots, \gamma_{T-1}\right)$ are the optimal stochastic controls to be determined in the solution of the stochastic control model of the GLWB. We also assume that payment to the beneficiary upon death of the policyholder occurring within $(i-1, i]$ will be paid at year $i$. In this case, the policy contract terminates and there will be no withdrawal at $i, i+1, \ldots, T-1$. Let $\mathcal{E}$ denote the set of admissible controls, where $\Gamma=\left(\gamma_{1}, \gamma_{2}, \ldots, \gamma_{T-1}\right) \in \mathcal{E}$. We consider an adapted control process $\Gamma: \Omega \times \mathcal{T} \rightarrow E$, where $E$ is measurable with respect to the filtered probability space $(\Omega, \mathcal{F}, Q)$. The bonus (roll-up) feature encourages the policyholder to choose zero withdrawal in order to take advantage of the proportional growth of the benefit base as bonus. From the issuer's perspective, the reduction of withdrawal amount leads to a lower chance of "ruined" where $W_{t}$ drops to zero value. Therefore, the withdrawal amount $\gamma_{i}$ at year $i$ may take value from zero up to the maximum of the policy fund value (corresponding to complete surrender). The policy fund value will be depleted by $\gamma_{i}$ upon withdrawal of amount $\gamma_{i}$ at year $i$, so

$$
W_{i}=W_{i^{-}}-\gamma_{i}, \quad 0 \leq \gamma_{i} \leq \max \left(W_{i^{-}}, G A_{i^{-}}\right)
$$

Note that even when $W_{i^{-}}$is lower than $G A_{i^{-}}$, the policyholder is entitled to withdraw $G A_{i^{-}}$as guaranteed by the provision in GLWB. Complete surrender corresponds to $\gamma_{i}=W_{i^{-}}$, where $W_{i^{-}}>G A_{i^{-}}$. Besides the death event, the surrender event is the other scenario that leads to termination of the policy contract.

For an excessive withdrawal beyond the contractual amount $G A_{i^{-}}$at year $i$, proportional penalty charge $k_{i}$ is applied on $\gamma_{i}-G A_{i}$, so the cash flow $f_{i}\left(\gamma_{i} ; A_{i^{-}}\right)$received by the policyholder at year $i$ is given by

$$
f_{i}\left(\gamma_{i} ; A_{i^{-}}\right)= \begin{cases}\gamma_{i} & \text { if } 0 \leq \gamma_{i} \leq G A_{i^{-}} \\ G A_{i^{-}}+\left(1-k_{i}\right)\left(\gamma_{i}-G A_{i^{-}}\right) & \text {if } G A_{i^{-}}<\gamma_{i} \leq W_{i^{-}}\end{cases}
$$

As a summary, $\gamma_{i}$ is capped by $\max \left(W_{i^{-}}, G A_{i^{-}}\right)$. That is, the policyholder is entitled to withdraw up to $G A_{i^{-}}$ when $W_{i^{-}} \leq G A_{i^{-}}$while the withdrawal amount is capped by $W_{i^{-}}$when $W_{i^{-}}>G A_{i^{-}}$.

The benefit base $A_{i}$ at year $i$ may be increased either by the roll-up feature or step-up feature. Suppose the policyholder chooses not to withdraw at year $i$, then the benefit base is increased proportionally by the bonus rate $b_{i}$, where

$$
A_{i}=A_{i^{-}}\left(1+b_{i}\right) \quad \text { if } \gamma_{i}=0 .
$$

When $\gamma_{i}$ is larger than $G A_{i^{-}}$, where $G A_{i^{-}}<\gamma_{i} \leq W_{i^{-}}$, not only there will be a proportional penalty charge on

the excess withdrawal amount $\gamma_{i}-G A_{i^{-}}$, the guarantee base is reduced by the factor $\frac{W_{i^{-}}-\gamma_{i}}{W_{i^{-}}-G A_{i^{-}}}$. In particular, when $\gamma_{i}=W_{i^{-}}$, the benefit base is reduced to zero. This corresponds to complete surrender. 
On the other hand, when $\gamma_{i}>0$, the ratchet provision (step-up) is activated on a ratchet date when the benefit base $A_{i}$ stays below $W_{i}=\left(W_{i^{-}}-\gamma_{i}\right)^{+}$after withdrawal of $\gamma_{i}$, where $x^{+}$denotes $\max (x, 0)$. Under this scenario, the benefit base $A_{i}$ is adjusted upward to $W_{i^{-}}-\gamma_{i}$ right after withdrawal of $\gamma_{i}$. The jump condition on the benefit base arising from the ratchet provision on a ratchet date is given by

$$
A_{i}= \begin{cases}\max \left(A_{i^{-}},\left(W_{i^{-}}-\gamma_{i}\right)^{+} \mathbf{1}_{\left\{i \in \mathcal{T}_{e}\right\}}\right) & \text { if } 0<\gamma_{i} \leq G A_{i^{-}} \\ \max \left(\frac{W_{i^{-}}-\gamma_{i}}{W_{i^{-}}-G A_{i^{-}}} A_{i^{-}},\left(W_{i^{-}}-\gamma_{i}\right) \mathbf{1}_{\left\{i \in \mathcal{T}_{e}\right\}}\right) & \text { if } G A_{i^{-}}<\gamma_{i} \leq W_{i^{-}}\end{cases}
$$

In summary, the values of $W_{i}$ and $A_{i}$ right after time $i$ have dependence on their values $W_{i^{-}}$and $A_{i^{-}}$ right before time $i$ and the control withdrawal amount $\gamma_{i}$, We define the vector function $\mathbf{h}\left(W_{i^{-}}, A_{i^{-}} ; \gamma_{i}\right)$ that characterizes the jump conditions due to withdrawal and either the roll-up (bonus) or step-up (ratchet) feature across time $i$ as follows:

$$
\begin{aligned}
& \left(\begin{array}{c}
W_{i} \\
A_{i}
\end{array}\right)=\mathbf{h}\left(W_{i^{-}}, A_{i^{-}} ; \gamma_{i}\right)
\end{aligned}
$$

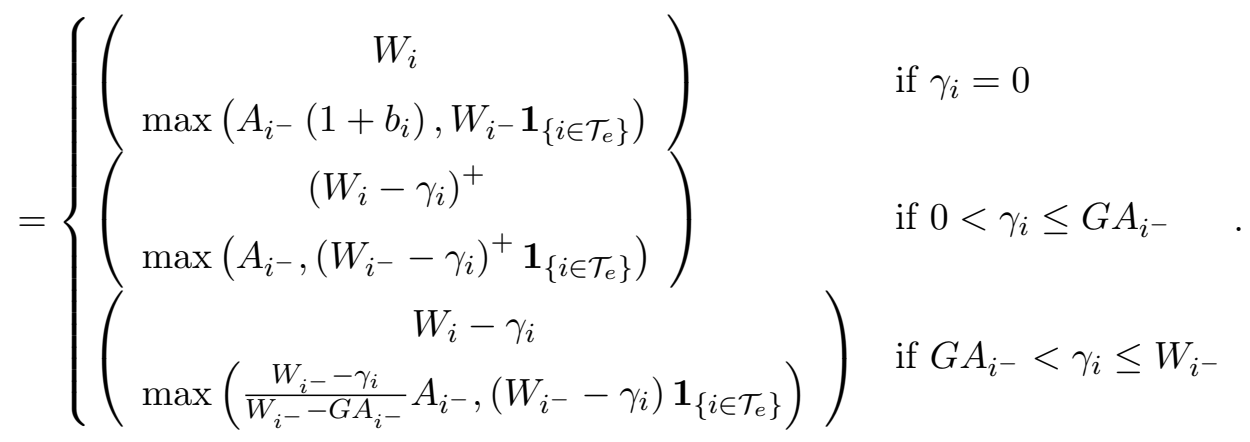

Note that the benefit base process $A_{t}$ remains constant over $[i, i+1)$ since there is no updating of the benefit base over the period. On the other hand, the fund value process $W_{t}$ fluctuates over $[i, i+1)$ according to the assumed stochastic volatility dynamics. It is then convenient to normalize the fund value process $W_{t}$ over $[i, i+1)$ by $A_{i}$ in our later Monte Carlo simulation calculations.

The value function $V(W, A, i)$ decreases by the net amount received by the policyholder $f_{i}\left(\gamma_{i} ; A_{i^{-}}\right)$across the withdrawal date $i$ when $\gamma_{i}>0$. The two state variables $W$ and $A$ in the value function $V$ would have jumps across a withdrawal date as well.

\section{Terminal conditions of the value function upon death and surrender events}

Suppose the payment to the beneficiary occurs at year $i$, the policy contract terminates. Correspondingly, there will be no withdrawal, so $\gamma_{i}=0$. The termination value of the policy on the death payment date $i$ is given by

$$
V(W, A, v, i)=W
$$

The policy also terminates at year $i$ when complete surrender occurs, where $\gamma_{i}=W_{i^{-}}>G A_{i^{-}}$. The value function on the surrender date $i$ is equal to $G A_{i^{-}}+\left(1-k_{i}\right)\left(W_{i^{-}}-G A_{i^{-}}\right)$, which is the withdrawal amount net of proportional penalty charge $k_{i}$ applied on $W_{i^{-}}-G A_{i^{-}}$. 


\section{Mathematical formulation of the value function}

We let $\tau$ be $\mathcal{F}$-stopping time taking values in the set $\mathcal{T}$ at which the policyholder chooses optimally to surrender the policy contract, that is,

$$
\tau=\inf \left\{i \in \mathcal{T} \mid \gamma_{i}=W_{i^{-}}>G A_{i^{-}}\right\} .
$$

Following the usual notation, we take $\tau=\infty$ if the policyholder dies before the surrender event occurs.

Conditional on a given control process $\Gamma$, the value function of the contract at time 0 is given by the risk neutral expectation of the discounted cash flows received by the policyholder. The value function $V(W, A, 0)$ at initiation is then given by taking the supremum among $\Gamma$ chosen within the admissible strategy set $\mathcal{E}$. In other words, we have

$$
V(W, A, v, 0)=\sup _{\Gamma \in \mathcal{E}} E_{Q}\left[\sum_{i=1}^{\tau \wedge(T-1)} e^{-r i}\left[p_{i} f_{i}\left(\gamma_{i} ; A_{i^{-}}\right)+p_{i-1} q_{i-1} W_{i^{-}}\right]+\mathbf{1}_{\{\tau>T-1\}} e^{-r T} p_{T-1} W_{T}\right] .
$$

Note that the stopping time $\tau$ would be specified once the optimal control process $\Gamma$ has been chosen.

\section{Regression-based Monte Carlo simulation algorithms}

The numerical solution of the stochastic control model of the GLWB poses mathematical challenges due to high dimensionality of the model, complex path dependence on the underlying fund value process due to withdrawal and ratchet / bonus events, and search of the optimal stochastic control on withdrawal strategies. The finite difference solution approach faces with the curse of dimensionality, which may be alleviated with some simplifying assumption on the dynamics of the policy fund process. For example, Forsyth and Vetzal (2014) use the finite difference scheme for pricing GLWB under the regime switching model. In recent years, the regression-based Monte Carlo simulation algorithms have been commonly adopted in pricing life insurance contracts and variable annuities with early surrender feature (Andreatta and Corradin, 2003; Bacinello et al., 2010). The regression-based Monte Carlo simulation algorithms (Longstaff and Schwartz, 2001; Tsitsiklis and Van Roy, 2001) for solving American option models are directly relevant since the early surrender feature resembles closely to the American early exercise feature. We show how to extend the regression-based Monte Carlo simulation algorithms to pricing stochastic control models of GLWB. The ease of implementation is facilitated by the bang-bang analysis of the strategy space of optimal withdrawal policies, which reduces the optimal withdrawal policies to the three choices: zero withdrawal, withdrawal at the contractual guaranteed amount or complete surrender.

First of all, we would like to formulate the dynamic programming procedure over successive time intervals $[i, i+1), i=0,1, \ldots, T-1$. The main challenge is the determination of the control process $\Gamma=\left(\gamma_{1}, \gamma_{2}, \ldots, \gamma_{T-1}\right)$ as part of the solution of the stochastic control model. We compute $V(W, A, v, 0)$ recursively by the following 
dynamic programming procedure

$$
\begin{aligned}
V\left(W, A, v, T^{-}\right)= & p_{T-1} W_{T} \\
V\left(W, A, v, i^{-}\right)= & p_{i-1} q_{i-1} W_{i^{-}}+p_{i} \sup _{\gamma_{i} \in\left[0, \max \left(W_{i^{-}}, G A_{i^{-}}\right)\right]}\left\{f_{i}\left(\gamma_{i} ; A_{i^{-}}\right)\right. \\
& \left.+e^{-r} E_{Q}\left[V\left(W, A, v,(i+1)^{-}\right) \mid\left(\begin{array}{c}
W_{i} \\
A_{i}
\end{array}\right)=\mathbf{h}\left(W_{i^{-}}, A_{i^{-}} ; \gamma_{i}\right), v_{i}=v\right]\right\}, i=1,2, \ldots, T-1,
\end{aligned}
$$

$$
V(W, A, v, 0)=e^{-r} E_{Q}\left[V\left(W, A, v, 1^{-}\right)\right]
$$

In the dynamic programming procedure over $[i, i+1), i=1,2, \ldots, T-1$, it is required to determine $\gamma_{i} \in\left[0, \max \left(W_{i^{-}}, G A_{i^{-}}\right)\right]$such that the sum of the cash flow $f_{i}\left(\gamma_{i} ; A_{i^{-}}\right)$and the conditional expectation

$e^{-r} E_{Q}\left[V\left(W, A, v,(i+1)^{-}\right) \mid\left(\begin{array}{c}W_{i} \\ A_{i}\end{array}\right)=\mathbf{h}\left(W_{i^{-}}, A_{i^{-}} ; \gamma_{i}\right), v_{i}=v\right]$ is maximized. Indeed, one has to face with the challenge of finding the optimal control $\gamma_{i}$ at time $i, i=1,2, \ldots, T-1$. In our Monte Carlo simulation algorithm, the conditional expectation under the optimal choice of $\gamma_{i}$ is estimated using the regression-based algorithm, the details of which are presented below. The success in the implementation of the least squares regression technique (Longstaff and Schwartz, 2001; Tsitsiklis and Van Roy, 2001) relies on the simplication of the strategy space of withdrawal policies to the three choices: $\gamma_{i}=0$ (zero withdrawal), $\gamma_{i}=G A_{i^{-}}$(contractual guaranteed amount) and $\gamma_{i}=W_{i^{-}}$(complete surrender).

The presentation of our regression-based Monte Carlo simulation algorithm is organized as follows. First, we establish the proposition on the bang-bang analysis of the strategy space of withdrawal policies of the GLWB model. We then present the discretized schemes for the simulation of the fund value process under the two stochastic volatility models: ONE model and 3/2-model. The main ingredient of our Monte Carlo algorithm is the least squares regression procedure in the determination of the optimal withdrawal control variables and the conditional expectation of the value function. It is observed that a high proportion of the simulation paths of the fund value process result in zero fund value before termination. By taking advantage that the "zero" state is an absorbing state for the fund value, we manage to obtain the closed form solution for the value function when the fund value hits the zero value. This would save the simulation time in the Monte Carlo calculations quite substantially. Lastly, we establish the convergence proof of our Monte Carlo simulation algorithm.

\subsection{Bang-bang analysis of the strategy space of withdrawal policies}

Azimzadeh and Forsyth (2014) apply the bang-bang analysis to establish that the strategy space of discrete withdrawal policies of the GLWB model under the regime switching framework is limited to either zero withdrawal, withdrawal of contractual amount and complete surrender. Under the assumed stochastic volatility dynamics for the underlying fund value process in our GLWB model, according to Corollary 4.2 in Azimzadeh and Forsyth (2014), the existence of a similar set of optimal bang-bang control policies is ensured provided that the European option price function preserves convexity. That is, for any convex terminal payoff function, the corresponding European option price would be always convex in the underlying asset price.

By applying the comparison results for stochastic volatility models via the coupling technique presented in Hobson (2010), we can establish the convexity preservation property of the European option price function for 
the 3/2-model, the details of which are stated in Proposition 1. By following a similar approach, one may also establish convexity preservation property for the Heston model and ONE model.

\section{Proposition 1 - Convexity of European price function under the 3/2-model}

Suppose the dynamics of $W_{t}$ and $v_{t}$ under the filtered probability space $(\Omega, \mathcal{F}, Q)$ is governed by the following 3/2-stochastic volatility model

$$
\begin{aligned}
d W_{t} & =(r-\eta) W_{t} d t+\sqrt{v_{t}} W_{t} d B_{t}^{W} \\
d v_{t} & =\kappa v_{t}\left(\theta-v_{t}\right) d t+\epsilon v_{t}^{\frac{3}{2}} d B_{t}^{v}
\end{aligned}
$$

where $d B_{t}^{W} d B_{t}^{v}=\rho d t$. For any convex terminal payoff function $\Phi\left(W_{T}\right)$, the corresponding time-0 European price function as given by

$$
\phi(w, v)=e^{-r T} E_{Q}\left[\Phi\left(W_{T}\right) \mid W_{0}=w, v_{0}=v\right]
$$

is also convex.

The proof of Proposition 1 is presented in Appendix A. Once the strategy space of the optimal withdrawal policies is only limited to three choices, it becomes computationally plausible to adopt the regression based Monte Carlo simulation. On each withdrawal date, the estimated continuation value obtained from the least squares regression is compared with the complete surrender value and the bonus value under zero withdrawal. In the dynamic programming procedure, the maximum value among these three values is taken.

\subsection{Closed form solution for the value function at zero fund value}

When a simulated path of the fund value process happens to correspond to the scenario of non-performance of the fund, the fund value may hit the zero value at some time prior to maturity $T$. Based on the bangbang analysis, the strategy space of withdrawal policies at zero fund value is limited to either zero withdrawal or withdrawal of contractual amount $G A$ since the choice of complete surrender is ruled out. Intuitively, the optimal withdrawal policies strike the balance between the growth of the benefit base, time value of the contractual withdrawals received and the mortality distribution. We let $V_{0}(A, t)$ denote the time- $t$ value function of the GLWB at zero fund value, where $W=0$. Let $i_{0}$ be the next withdrawal date right after time $t$, where $i_{0}=\inf \{i: i>t\}$. It is obvious that

$$
V_{0}(A, t)=0 \quad \text { when } i_{0}=T
$$

since there will be no more guaranteed withdrawal beyond $t$. Next, when $i_{0}=T-1$, the policyholder should withdraw the contractual amount $G A$ at $i_{0}$ since this would be the last guaranteed withdrawal before maturity. Therefore, we have

$$
V_{0}(A, t)=e^{-r(T-1-t)} p_{T-1} G A \quad \text { when } i_{0}=T-1 .
$$

To determine $V_{0}(A, t)$ for $i_{0} \leq T-2$, it is necessary to characterize the optimal withdrawal policies on the future successive withdrawal dates $\left\{i_{0}, i_{0}+1, \ldots, T-1\right\}$ beyond $t$. 
The strategy $\gamma=0$ means forfeiture of $G A$ on the withdrawal date in return for an increment of the benefit base that would generate higher contractual withdrawals on later withdrawal dates. Therefore, the zero withdrawal strategy would be adopted on earlier withdrawal dates while withdrawal of contractual amount would be adopted on later withdrawal dates closer to maturity. Indeed, we can establish the following optimal withdrawal policies under $W=0$. There exists $i_{G}^{*}$ such that the optimal withdrawal amount on withdrawal date $i$ is given by

$$
\gamma_{i}= \begin{cases}0 & i_{0} \leq i<i_{G}^{*} \\ G A_{i} & i_{G}^{*} \leq i \leq T-1\end{cases}
$$

where $i_{G}^{*}$ assumes some value between $i_{0}$ and $T-1$, inclusively. The value function $V_{0}(A, t)$ is determined by choosing $i_{G}^{*}$ that maximizes the sum of present value of all future withdrawals based on the optimal withdrawal policies. The analytic representation of $V_{0}(A, t)$ for $i_{0} \leq T-2$ is presented in Proposition 2 .

\section{Proposition 2}

Given that the bonus rate $b_{i}$ is non-increasing in $i$ and the death probability $q_{i}$ is non-decreasing in $i$. The time- $t$ value function $V_{0}(A, t)$ of the GLWB at zero fund value is given by

$$
V_{0}(A, t)=\max _{i_{G}^{*} \in\left\{i_{0}, i_{0}+1, \ldots, T-1\right\}}\left\{\prod_{i=i_{0}}^{i_{G}^{*}-1}\left(1+b_{i}\right)\left[\sum_{k=i_{G}^{*}}^{T-1} e^{-r(k-t)} p_{k}\right]\right\} G A
$$

for $i_{0} \leq T-2$, where $i_{0}=\inf \{i: i>t\}$.

The proof of Proposition 2 is presented in Appendix B.

\subsection{Regression-based Monte Carlo algorithm}

First, we would like to comment on the generation of Monte Carlo simulation paths of the fund value process under the 3/2-model and ONE model. Unlike the Heston model, the literature on the simulation of Monte Carlo paths under the 3/2-model and ONE model has been relatively thin. Baldeaux (2012) proposes the exact simulation of the 3/2-model as an extension of the Broadie-Kaya (2006) exact simulation algorithm for the Heston model. However, the computational effort in the evaluation of the distribution of the conditional integrated variance is quite involved (requiring numerical evaluation of the modified Bessel functions). In terms of overall computational efficiency and ease of computation, we find that the exact simulation algorithm may not compete favorably well with the Euler scheme in the generation of simulation paths under the $3 / 2$ model and ONE model. It is commonly known that the Euler discretization of the Heston model may have a positive probability of generating negative value for the stochastic variance process. Lord et al. (2010) propose a modified Euler-type scheme with special treatment for the negative variance. Their extensive numerical experiments demonstrate that their Euler scheme outperform all other discretization schemes, including the exact simulation algorithm of Broadie and Kaya (2006). In our numerical calculations of generating the Monte Carlo simulation paths, we use the Lord's modified Euler scheme for the ONE model. By adopting the logtransformed variance variable in the 3/2-model, the nuisance of occurrence of negative variance value would not occur. Hence, the usual Euler scheme is used for the path simulation of the $3 / 2$-model in our calculations. 


\subsubsection{Euler discretized schemes}

Under the filtered probability space $(\Omega, \mathcal{F}, Q)$, the ONE model assumes the following dynamics

$$
\begin{aligned}
d W_{t} & =(r-\eta) W_{t} d t+\sqrt{v_{t}} W_{t} d B_{t}^{W} \\
d v_{t} & =\kappa\left(\theta-v_{t}\right) d t+\epsilon v_{t} d B_{t}^{v},
\end{aligned}
$$

where $d B_{t}^{W} d B_{t}^{v}=\rho d t$. We let $\hat{W}_{j}$ and $\hat{v}_{j}$ denote the time-discretized approximation to $W_{t}$ and $v_{t}$ at time $j h$, respectively, where $h>0$ is the constant time step. Starting with $\hat{W}_{0}=W_{0}$ and $\hat{v}_{0}=v_{0}$, based on the Lord's approach of ensuring non-negative value for the discretized variance, the modified Euler discretization of the ONE model is constructed as follows

$$
\begin{aligned}
\hat{W}_{j+1} & =\hat{W}_{j} \exp \left\{\left(r-\eta-\frac{\hat{v}_{j}}{2}\right) h+\sqrt{\hat{v}_{j} h} Z_{j+1}^{W}\right\} \\
\tilde{v}_{j+1} & =\tilde{v}_{j}+\kappa\left(\theta-\tilde{v}_{j}^{+}\right) h+\epsilon \tilde{v}_{j}^{+} \sqrt{h} Z_{j+1}^{v} \\
\hat{v}_{j+1} & =\tilde{v}_{j+1}^{+}, \quad j=0,1, \ldots
\end{aligned}
$$

Here, $\left(Z_{1}^{W}, Z_{1}^{v}\right),\left(Z_{2}^{W}, Z_{2}^{v}\right), \ldots$, are independent two-dimensional vectors of standard normal random variables, where $Z_{j}^{W}$ and $Z_{j}^{v}$ are correlated with correlation coefficient $\rho_{j}, j=0,1, \ldots$ More specifically, we have the usual Euler scheme when $\tilde{v}_{j}$ is non-negative. When $\tilde{v}_{j}$ falls below zero, then it increases at the drift rate $\kappa \theta$ over the time interval $h$. This gives

$$
\tilde{v}_{j+1}=\tilde{v}_{j}+\kappa \theta h \quad \text { and } \quad \hat{v}_{j+1}=\tilde{v}_{j+1}^{+}
$$

For the 3/2-model defined in eqs. (3.2a,b), we take the log-transformation of $W_{t}$ and $v_{t}$ so that the governing stochastic differential equations for $\ln W_{t}$ and $\ln v_{t}$ become

$$
\begin{aligned}
d \ln W_{t} & =\left(r-\eta-\frac{v_{t}}{2}\right) d t+\sqrt{v_{t}} d B_{t}^{W} \\
d \ln v_{t} & =\tilde{\kappa}\left(\tilde{\theta}-v_{t}\right) d t+\epsilon \sqrt{v_{t}} d B_{t}^{v}
\end{aligned}
$$

where $\tilde{\kappa}=\kappa+\frac{\epsilon^{2}}{2}$ and $\tilde{\theta}=\frac{\kappa \theta}{\kappa+\frac{\epsilon^{2}}{2}}$. The log-transformation ensures positivity of $W_{t}$ and $v_{t}$ so that the usual Euler discretization can be applied. Starting with $\hat{W}_{0}=W_{0}$ and $\hat{v}_{0}=v_{0}$, the Euler scheme for the 3/2-model is given by

$$
\begin{aligned}
\hat{W}_{j+1} & =\hat{W}_{j} \exp \left\{\left(r-\eta-\frac{v_{j}}{2}\right) h+\sqrt{\hat{v}_{j} h} Z_{j+1}^{W}\right\} \\
\hat{v}_{j+1} & =\hat{v}_{j} \exp \left\{\tilde{\kappa}\left(\tilde{\theta}-\hat{v}_{j}\right) h+\epsilon \sqrt{\hat{v}_{j} h} Z_{j+1}^{v}\right\}, \quad j=0,1, \ldots
\end{aligned}
$$

\subsubsection{Three-step regression-based algorithm}

The core of the least squares algorithm consists of three key steps. In the first step, we perform simulation runs for the paths of the policy fund value, benefit base and variance by presetting the control on the withdrawal to be the contractual withdrawal amount on all withdrawal dates. This first step serves to generate simulating paths for the later conditional expectation calculations. This is similar to the least squares Monte Carlo algorithm 
for valuation of an American option where simulation paths are first generated by assuming "continuation" on each exercise date. The optimal strategy is later determined using the dynamic programming principle. Since the benefit base $A_{i}$ stays constant over $[i, i+1), i=0,1, \ldots, T-1$, and thanks to the homogeneity property of the value function, we achieve dimension reduction by normalizing the value function at $(i+1)^{-}$ and the state variables at $i$ by the constant benefit base $A_{i}$ for each interval $[i, i+1), i=0,1, \ldots, T-1$. In the second step, we approximate the conditional expectation of the normalized value function by regressing between the normalized value function at $(i+1)^{-}$and the normalized fund value and variance at $i$. In the third step, we recover the original conditional expectation by multiplying the benefit base $A_{i}$, then determine the optimal strategy and the value function at $i^{-}$by incorporating the jump conditions at time $i$ and exerting the bang-bang analysis of the strategy space. The last two steps constitute a recursive procedure, starting with the initiation of the known terminal payoff at time $T$.

The details of the last two steps are presented as follows. Let $h_{1}\left(W_{i^{-}}, A_{i^{-}} ; \gamma_{i}\right)$ and $h_{2}\left(W_{i^{-}}, A_{i^{-}} ; \gamma_{i}\right)$ denote the first and second component of $\mathbf{h}\left(W_{i^{-}}, A_{i^{-}} ; \gamma_{i}\right)$, respectively. Note that the benefit base $A_{i}$ is simply equal to the second component $h_{2}\left(W_{i^{-}}, A_{i^{-}} ; \gamma_{i}\right)$, which stays at the same value over $[i, i+1)$. Recall that in the conditional expectation calculations over successive time intervals, we take advantage of the homogeneity property of the price function of GLWB with respect to $A_{i}$. We normalize $W_{t}$ and $V(W, A, v, t)$ by $A_{i}$ as follows

$$
\widetilde{W}_{t}=W_{t} / A_{i} \quad \text { and } \quad \widetilde{V}(\widetilde{W}, v, t)=V(W, A, v, t) / A_{i}, \quad t \in[i, i+1) .
$$

After normalizing the value function and fund value by $A_{i}$ over $[i, i+1), i=1,2, \ldots, T-1$, eq. (3.1) becomes

$$
\begin{aligned}
V\left(W, A, v, i^{-}\right)= & p_{i-1} q_{i-1} W_{i^{-}}+p_{i} \sup _{\gamma_{i}}\left\{f_{i}\left(\gamma_{i} ; A_{i^{-}}\right)\right. \\
& \left.+e^{-r} A_{i} E_{Q}\left[\widetilde{V}\left(\widetilde{W}, v,(i+1)^{-}\right) \mid \widetilde{W}_{i}=\widetilde{h}_{1}\left(W_{i^{-}}, A_{i^{-}} ; \gamma_{i}\right), v_{i}=v\right]\right\}, \quad i=1,2, \ldots, T-1,
\end{aligned}
$$

where $\widetilde{h}_{1}\left(W_{i^{-}}, A_{i^{-}} ; \gamma_{i}\right)$ is defined by $h_{1}\left(W_{i^{-}}, A_{i^{-}} ; \gamma_{i}\right) / A_{i}$. By virtue of the bang-bang analysis of the strategy space, we can limit the search for the optimal $\gamma_{i}$ to only three choices so that the above equation reduces to

$$
\begin{aligned}
& V\left(W, A, v, i^{-}\right)= p_{i-1} q_{i-1} W_{i^{-}}+p_{i} \max _{\gamma_{i} \in\left\{0, G A_{i^{-}}, W_{i^{-}}\right\}}\left\{f_{i}\left(\gamma_{i} ; A_{i^{-}}\right)\right. \\
&\left.+e^{-r} A_{i} E_{Q}\left[\widetilde{V}\left(\widetilde{W}, v,(i+1)^{-}\right) \mid \widetilde{W}_{i}=\widetilde{h}_{1}\left(W_{i^{-}}, A_{i^{-}} ; \gamma_{i}\right), v_{i}=v\right]\right\}, \\
& i=1,2, \ldots, T-1 .
\end{aligned}
$$

The next step is to approximate the conditional expectation in eq. (3.8) by regression, where the regression takes the form

$$
E_{Q}\left[\widetilde{V}\left(\widetilde{W}, v,(i+1)^{-}\right) \mid \widetilde{W}_{i}=\widetilde{W}, v_{i}=v\right] \approx \sum_{j=1}^{J} \beta_{i j}^{(J)} \psi_{j}(\widetilde{W}, v)=\Psi(\widetilde{W}, v) \boldsymbol{\beta}_{i}^{(J)}
$$

Here,

$$
\Psi(\widetilde{W}, v)=\left(\psi_{1}(\widetilde{W}, v), \ldots, \psi_{J}(\widetilde{W}, v)\right)
$$


is the vector of basis functions and

$$
\boldsymbol{\beta}_{i}^{(J)}=\left(\beta_{i 1}^{(J)}, \beta_{i 2}^{(J)}, \ldots, \beta_{i J}^{(J)}\right)^{T}
$$

is the vector of the regression coefficients. The basis functions adopted in the our algorithm are chosen to be the Laguerre polynomials with the damping factor $e^{-\frac{x}{5}}$. The multiplication of the damping factor avoids the occurrence of exceedingly large values in the basis functions. We use the superscript $m$ to denote the $m^{\text {th }}$ realized path and $M$ to denote the total number of sample paths. For a given fixed value of $M$, we then solve for the vector of regression coefficients $\boldsymbol{\beta}_{i}^{(M, J)}$ by the following least squares regression

$$
\boldsymbol{\beta}_{i}^{(M, J)}=\underset{\boldsymbol{\beta}_{i} \in R^{J}}{\arg \min } \sum_{m=1}^{M}\left[\Psi\left(\widetilde{W}_{i}^{(m)}, v_{i}^{(m)}\right) \boldsymbol{\beta}_{i}-\widetilde{V}\left(\widetilde{W}^{(m)}, v^{(m)},(i+1)^{-}\right)\right]^{2} .
$$

In the numerical procedure, the true value $\widetilde{V}\left(\widetilde{W}^{(m)}, v^{(m)},(i+1)^{-}\right)$is unknown and has to be replaced by the estimated value $\widetilde{V}\left(\widetilde{W}^{(m)}, v^{(m)},(i+1)^{-} ; \boldsymbol{\beta}_{i+1}^{(M, J)}\right)$, where the conditional expectation is approximated by the regressed value based on the regression with coefficients $\boldsymbol{\beta}_{i+1}^{(M, J)}$. To initiate the recursive algorithm, $\widetilde{V}\left(\widetilde{W}^{(m)}, v^{(m)}, T^{-}\right)$is set to be the known normalized terminal payoff.

Once the regression coefficients $\boldsymbol{\beta}_{i}^{(M, J)}$ have been obtained, the optimal control $\gamma_{i}^{*}$ can be found by comparing the three decision choices $\left\{0, G A_{i^{-}}, W_{i^{-}}\right\}$in eq. (3.8). In terms of the optimal control $\gamma_{i}^{*}$, we have

$$
\begin{aligned}
V\left(W^{(m)}, A^{(m)}, v^{(m)}, i^{-} ; \boldsymbol{\beta}_{i}^{(M, J)}\right)= & p_{i-1} q_{i-1} W_{i^{-}}^{(m)}+p_{i}\left[f_{i}\left(\gamma_{i}^{*} ; A_{i^{-}}^{(m)}\right)\right. \\
& \left.+e^{-r} A_{i}^{(m)} \Psi\left(\tilde{h}_{1}\left(W_{i^{-}}^{(m)}, A_{i^{-}}^{(m)} ; \gamma_{i}^{*}\right), v^{(m)}\right) \boldsymbol{\beta}_{i}^{(M, J)}\right] .
\end{aligned}
$$

We perform the above procedure in backward induction on $i$ to obtain the regressed coefficients $\boldsymbol{\beta}_{i}, i=$ $1,2, \ldots, T-1$. The final step would be the approximation of $V(W, A, v, 0)$ by the average of the discounted value of $V\left(W^{(m)}, A^{(m)}, v^{(m)}, 1^{-} ; \boldsymbol{\beta}_{1}^{(M, J)}\right)$.

When $\widetilde{W}_{i}$ is zero in eq. (3.8), we use the analytical solution instead of the regressed value for the conditional expectation. The use of the analytical solution of the value function when the fund value $W$ is zero can reduce the computation work in the Monte Carlo algorithm significantly and enhance accuracy of the algorithm. Also, the regression procedure in eq. (3.10) only involves those paths that have positive normalized fund value. By doing this, the approximation error due to the regression is reduced and the computation time required for the regression procedure is lowered. This follows the same spirit of regressing the in-the-money paths in Longstaff and Schwartz (2001).

We summarize the numerical procedure for the Least Squares Monte Carlo algorithm as follows.

\section{Least Squares Monte Carlo algorithm (LSMC algorithm)}

1. We preset the control process $\bar{\Gamma}$ to be contractual guaranteed withdrawal amount on each withdrawal date and simulate $M$ independent realized paths of the policy fund value, benefit base and variance as denoted by $\left\{\left(W_{1^{-}}, A_{1^{-}}, v_{1^{-}}\right)^{(m)},\left(W_{2^{-}}, A_{2^{-}}, v_{2^{-}}\right)^{(m)}, \ldots,\left(W_{T^{-}}, A_{T^{-}}, v_{T^{-}}\right)^{(m)}\right\}, m=1, \ldots, M$.

2. On the terminal date $T^{-}$, we set $V\left(W^{(m)}, A^{(m)}, v^{(m)}, T^{-}\right)=p_{T-1} W_{T^{-}}^{(m)}$.

3. We apply the backward induction procedure for $i=T-1, \ldots, 1$, as follows 
(a) Update the normalized fund value $\widetilde{W}_{i}^{(m)}$ and normalized payoff $\widetilde{V}\left(\widetilde{W}^{(m)}, v^{(m)},(i+1)^{-} ; \boldsymbol{\beta}_{i+1}^{(M, J)}\right)$ by

$$
\begin{aligned}
\widetilde{W}_{i}^{(m)} & =\tilde{h}_{1}\left(W_{i^{-}}^{(m)}, A_{i^{-}}^{(m)} ; \bar{\gamma}_{i}\right), \\
A_{i}^{(m)} & =h_{2}\left(W_{i^{-}}^{(m)}, A_{i^{-}}^{(m)} ; \bar{\gamma}_{i}\right), \\
\widetilde{V}\left(\widetilde{W}^{(m)}, v^{(m)},(i+1)^{-} ; \boldsymbol{\beta}_{i+1}^{(M, J)}\right) & =V\left(W^{(m)}, A^{(m)}, v^{(m)},(i+1)^{-} ; \boldsymbol{\beta}_{i+1}^{(M, J)}\right) / A_{i}^{(m)} .
\end{aligned}
$$

For $i=T-1$, we use

$$
V\left(W^{(m)}, A^{(m)}, v^{(m)}, T^{-}\right)=p_{T-1} W_{T^{-}}^{(m)}
$$

for the value of $V\left(W^{(m)}, A^{(m)}, v^{(m)},(i+1)^{-} ; \boldsymbol{\beta}_{i+1}^{(M, J)}\right)$.

(b) Using the paths that have positive value of $\widetilde{W}_{i}^{(m)}$, we determine $\boldsymbol{\beta}_{i}^{(M, J)}$ by the regression procedure shown in eq. (3.10).

(c) For the paths that have positive value of $W_{i^{-}}^{(m)}$, we apply the dynamic programming procedure of comparing the three values of the following payoff

$$
p_{i-1} q_{i-1} W_{i^{-}}^{(m)}+p_{i}\left[f_{i}\left(\gamma_{i} ; A_{i^{-}}^{(m)}\right)+e^{-r} A_{i}^{(m)} \Psi\left(\tilde{h}_{1}\left(W_{i^{-}}^{(m)}, A_{i^{-}}^{(m)} ; \gamma_{i}\right), v^{(m)}\right) \boldsymbol{\beta}_{i}^{(M, J)}\right],
$$

corresponding to $\gamma_{i}$ taking either $0, G A_{i^{-}}$or $W_{i^{-}}$. We set $V\left(W^{(m)}, A^{(m)}, v^{(m)}, i^{-} ; \boldsymbol{\beta}_{i}^{(M, J)}\right)$ to be the maximum value among the three payoffs. Note that $\Psi\left(\tilde{h}_{1}\left(W_{i^{-}}^{(m)}, A_{i^{-}}^{(m)} ; \gamma_{i}\right), v^{(m)}\right) \boldsymbol{\beta}_{i}^{(M, J)}$ is replaced by the analytical solution at zero policy fund value if $\tilde{h}_{1}\left(W_{i^{-}}^{(m)}, A_{i^{-}}^{(m)} ; \gamma_{i}\right)=0$.

(d) For the paths that have zero policy fund value $W_{i^{-}}^{(m)}$, we set $V\left(W^{(m)}, A^{(m)}, v^{(m)}, i^{-} ; \boldsymbol{\beta}_{i}^{(M, J)}\right)$ to be the analytical solution at zero fund value.

4. Lastly, we set $V(W, A, v, 0)=e^{-r} \frac{1}{M} \sum_{m=1}^{M} V\left(W^{(m)}, A^{(m)}, v^{(m)}, 1^{-} ; \boldsymbol{\beta}_{1}^{(M, J)}\right)$.

\section{Low-biased algorithm (LB algorithm)}

The estimated regression coefficients $\boldsymbol{\beta}_{i}^{(M, J)}$ determine the approximation of the conditional expectation for the given state of policy fund value and variance. The available regression coefficients would inform implicitly how the optimal withdrawal strategy can be adopted. By simulating a second set of Monte Carlo paths and applying the optimal withdrawal strategy deduced from the first-pass estimator, the value of the GLWB contract can be estimated. This would be biased low since no policy can be better than the optimal withdrawal strategy. The first-pass estimator based on the regression-based algorithm is often biased high (Glasserman, 2004). The spread between the high and low estimates provides a measure of accuracy of the least squares fitting achieved with the chosen set of basis functions.

\subsubsection{Convergence analysis of the Monte Carlo algorithm}

There are two distinct types of approximations involved in the Monte Carlo algorithms. First, approximation errors occur in the least squares regression procedure since we use a finite number of basis functions for the conditional expectation calculations in the dynamic programming procedure. Second, only a finite number of 
Monte Carlo simulation paths are employed for the estimation of the conditional expectation. The nonexact simulation of the state variables under the discretized schemes may introduce another type of approximation error. In our convergence proof of the Monte Carlo algorithm, we focus on the error analysis of the above two types of approximation by assuming that the state variables are simulated exactly (Broadie and Kaya, 2006; Baldeaux, 2012). Numerical tests verify that the errors caused by the discretized simulation schemes are generally quite small. One may refer to Higham and Mao (2005) and Lord et al. (2010) for a discussion of the error analysis arising from the discretization of the dynamic equations of the fund value process.

First, we prove that the error in the least squares regression procedure tends to zero as the number of basis functions goes to infinity. Second, given a fixed number of basis functions, the error in the estimation of conditional expectation from Monte Carlo simulation tends to zero as the number of simulation paths goes to infinity. For the sake of simplicity, our error analysis does not include the procedure where the closed form solution for the price function is used in the algorithm when the fund value becomes zero. The result of our convergence analysis should remain valid even with the inclusion of the closed form solution when the fund value becomes zero since no error is introduced by the exact closed form solution. Our proof differs from those in the literature in two aspects, namely, the stochastic control nature of the GLWB model and complexity in the jump conditions on each withdrawal date. There are related works on convergence analysis of the regression-based Monte Carlo simulation algorithms for solving optimal stopping problems. For example, Longstaff and Schwarz (2001), Tsitsiklis and Roy (2001) and Clément et al. (2002) present the convergence analysis of the relevant Monte Carlo simulation schemes in the context of pricing American options.

Under the preset control process $\bar{\Gamma}$, the risk neutral state process $\left\{\left(W_{t}, A_{t}, v_{t}\right) \mid 0 \leq t \leq T\right\}$ is a Markovian process and the stochastic volatility model preserves convexity, which then ensures the bang-bang analysis of the strategy space. Consider the time interval $[i, i+1)$, we let $\widetilde{\mathbf{X}}_{t}, i \leq t<i+1$, denote the vector stochastic process whose components are the normalized policy fund value $\widetilde{W}_{t}, i \leq t<i+1$, and stochastic variance $v_{t}$. Throughout the following discussion, we let $|\mathbf{x}|$ denote the Euclidean norm of a vector $\mathbf{x}$. We assume the set of basis functions $\Psi(\cdot)=\left\{\psi_{j}(\cdot)\right\}$ to satisfy the following conditions

H.1 : For $i=1,2, \ldots, T-1, \Psi\left(\widetilde{\mathbf{X}}_{i}\right)$ is total in $L^{2}\left(\sigma\left(\widetilde{\mathbf{X}}_{i}\right)\right)$; that is, $\operatorname{span} \Psi\left(\widetilde{\mathbf{X}}_{i}\right)=\left\{c_{1} \psi_{1}\left(\widetilde{\mathbf{X}}_{i}\right)+\cdots+c_{n} \psi_{n}\left(\widetilde{\mathbf{X}}_{i}\right): n \in \mathbb{N}, c_{1}, \ldots, c_{n} \in \mathbb{K}, \psi_{1}, \ldots, \psi_{n} \in \Psi\right\}$ is dense in $L^{2}\left(\sigma\left(\widetilde{\mathbf{X}}_{i}\right)\right)$.

H.2 : For $i=1,2, \ldots, T-1$ and $J \geq 1$, if $\sum_{k=1}^{J} \lambda_{k} \psi_{k}\left(\widetilde{X}_{i}\right)=0$ a.s., then $\lambda_{k}=0$ for $k=1,2, \ldots, J$.

The approximate value functions $V^{(M, J)}(W, A, v, 0)$ and $V^{(J)}(W, A, v, 0)$ are defined by

$$
V^{(M, J)}(W, A, v, 0)=e^{-r} \frac{1}{M} \sum_{m=1}^{M} V\left(W^{(m)}, A^{(m)}, v^{(m)}, 1^{-} ; \boldsymbol{\beta}_{1}^{(M, J)}\right),
$$

and

$$
V^{(J)}(W, A, v, 0)=e^{-r} E V\left(W, A, v, 1^{-} ; \boldsymbol{\beta}_{1}^{(J)}\right)
$$

respectively. In our two-step proof, we would like to prove that (i) for any fixed $J, V^{(M, J)}(W, A, v, 0)$ converges almost surely to $V^{(J)}(W, A, v, 0)$ as $M$ goes to infinity, (ii) and $V^{(J)}(W, A, v, 0)$ converges to $V(W, A, v, 0)$ as $J$ goes to infinity. 
As an important mathematical property in the construction of the proof, we would like to remark that under condition H.2, the $J$ dimensional least squares estimator $\boldsymbol{\beta}_{i}^{(J)}$ has the following explicit recursive identity: ${ }^{1}$

$$
\boldsymbol{\beta}_{i}^{(J)}=e^{-r}\left(R_{i}^{(J)}\right)^{-1} E\left[\widetilde{V}\left(\widetilde{X},(i+1)^{-} ; \boldsymbol{\beta}_{i+1}^{(J)}\right) \Psi^{(J)}\left(\widetilde{X}_{i}\right)\right]
$$

Here, $R_{i}^{(J)}$ is an $J \times J$ matrix, whose coefficients are given by

$$
\left(R_{i}^{(J)}\right)_{1 \leq k, l \leq J}=E\left[\psi_{k}\left(\tilde{X}_{i}\right) \psi_{l}\left(\tilde{X}_{i}\right)\right]
$$

\section{Proposition 3}

For $i=1,2, \ldots, T-1$, we have

$$
\lim _{J \rightarrow \infty} V\left(W, A, v, i^{-} ; \boldsymbol{\beta}_{i}^{(J)}\right)=V\left(W, A, v, i^{-}\right)
$$

in $L^{2}$.

The proof of Proposition 3 is presented in Appendix C. By letting the number of basis functions go to infinity, the direct consequence of Proposition 3 reveals that $V^{(J)}(W, A, v, 0)$ converges to $V(W, A, v, 0)$. For the remaining part, we would like to show that $V^{(M, J)}(W, A, v, 0)$ converges almost surely to $V^{(J)}(W, A, v, 0)$ as $M$ goes to infinity. The key steps in establishing the above results are summarized in Lemma 4 and Proposition 5.

\section{Lemma 4}

For $i=1,2, \ldots, T-1, \boldsymbol{\beta}_{i}^{(M, J)}$ converges almost surely to $\boldsymbol{\beta}_{i}^{(J)}$ as $M$ goes to infinity.

The proof of Lemma 4 is presented in Appendix D. Based on Lemma 4, it becomes readily to establish that $V^{(M, J)}(W, A, 0)$ converges almost surely to $V^{(J)}(W, A, 0)$ as $M$ goes to infinity.

\section{Proposition 5}

For $i=1,2, \ldots, T-1, V\left(W, A, i^{-} ; \boldsymbol{\beta}_{i}^{(M, J)}\right)$ converges almost surely to $V\left(W, A, i^{-} ; \boldsymbol{\beta}_{i}^{(J)}\right)$ as $M$ goes to infinity.

The proof of Proposition 5 is presented in Appendix E. Since

$$
V^{(M, J)}(W, A, v, 0)=\frac{1}{M} \sum_{m=1}^{M} e^{-r} V\left(W, A, 1^{-} ; \boldsymbol{\beta}_{1}^{(M, J)}\right)
$$

the result that $V^{(M, J)}(W, A, v, 0)$ converges almost surely to $V^{(J)}(W, A, v, 0)$ as $M$ goes to infinity is the direct consequence of Proposition 5 and the law of large numbers.

\footnotetext{
${ }^{1}$ For $i=T-1, \widetilde{V}\left(\widetilde{X}^{(m)},(i+1)^{-} ; \boldsymbol{\beta}_{i+1}^{(M, J)}\right)$ is replaced by the normalized terminal payoff, which does not depend on the regression estimator $\boldsymbol{\beta}$.
} 


\section{$4 \quad$ Numerical studies}

In this section, we would like to demonstrate the performance of the Least Squares Monte Carlo algorithm (abbreviated as LSMC) and the Low Biased algorithm (abbreviated as LB) for pricing the GLWB products under various contractual provisions and the two stochastic volatility models: 3/2-model and ONE model. To serve as a numerical validation, we compare the numerical results obtained from our algorithms with those obtained by the finite difference scheme in Forsyth and Vetzal (2014) under the Geometric Brownian motion assumption of the fund value process. We also conduct the sensitivity analysis of the GLWB price function by varying the model parameters in the stochastic volatility models and contractual features, as well as adopting sub-optimal withdrawal behavior of the policyholder. In addition, we examine the propensity of complete surrender under various ratchet clauses and penalty charges.

\subsection{Validation of numerical accuracy}

We compare numerical accuracy and computational efficiency of the LSMC and LB algorithms with the finite difference scheme in Forsyth and Vetzal (2014) in pricing the GLWB product under the assumption of the optimal withdrawal behavior of the policyholder. In order to set the stage for numerical comparison, we assume the dynamics of the underlying fund value to be governed by the Geometric Brownian motion, which is the same as that adopted by Forsyth and Vetzal (2014) in their single regime case. The model and contract parameters listed in Table 1 are taken as the "base" case in our numerical calculations.

\begin{tabular}{lc}
\hline Parameter & Value \\
\hline Volatility, $\sigma$ & 0.20 \\
Interest rate, $r$ & 0.04 \\
Penalty for excess withdrawal, $k(t)$ & $0 \leq t \leq 1: 3 \%, 1<t \leq 2: 2 \%$, \\
& $2 \leq t \leq 3: 1 \%, 3<t \leq 4: 0 \%$ \\
Expiry time, $T$ (years) & 57 \\
Initial payment, $S_{0}$ & 100 \\
Mortality & DAV 2004R (65 year old male) \\
& (Pasdika and Wolff, 2005) \\
Mortality payments & At year end \\
Withdrawal rate, $G$ & 0.05 annual \\
Bonus (no withdrawal) & 0.06 annual \\
Ratchet cycle & Every three years \\
Withdrawal strategy & Optimal \\
Withdrawal dates & yearly \\
\hline
\end{tabular}

Table 1: Model and contract parameters used in the numerical comparison with Forsyth and Vetzal (2014).

Using the LSMC and LB algorithms, we compute the value of the GLWB product under four different scenarios: "Base", "No bonus", "No surrender" and "No ratchet", Corresponding to Cases I, II, III, IV, respectively, in Table 2. The numerical results in the second column in Table 2 are obtained using the finite difference scheme in Forsyth and Vetzal (2014). To perform fair comparison in computation time and accuracy, we adopt the bang-bang strategy and homogeneity property in the finite difference scheme. We also utilize unequally spaced grid to enhance computational efficiency in the finite difference scheme. The bracket quantities in the second 
column are the insurance fee and CPU time. The third column lists the number of Laguerre basis functions used in the regression. In the finite difference scheme, we use 581 nodes to discretize $W$ and $A$ with 2280 steps in time discretization. We used $10^{5}$ simulation paths for the LSMC and LB algorithms. The standard deviation (SD) in the LSMC algorithm is seen to be smaller than that of the LB algorithm. Also, the numerical results are quite insensitive to the number of basis functions used in the regression calculations.

\begin{tabular}{|c|c|c|c|c|c|c|}
\hline \multirow{2}{*}{ Case } & \multirow{2}{*}{ Forsyth and Vetzal } & \multirow{2}{*}{ Number of basis functions } & \multicolumn{2}{|c|}{ LSMC } & \multicolumn{2}{|r|}{$\mathrm{LB}$} \\
\hline & & & price & $\mathrm{SD}(\mathrm{CPU})$ & price & $\mathrm{SD}(\mathrm{CPU})$ \\
\hline \multirow{3}{*}{ I } & \multirow{3}{*}{$100(149.3 \mathrm{bp}, 64.5 \mathrm{~s})$} & 6 & 100.0381 & $0.0414(5.1 \mathrm{~s})$ & 99.9924 & $0.0950(1.9 \mathrm{~s})$ \\
\hline & & 9 & 100.0412 & $0.0413(9.07 \mathrm{~s})$ & 99.9588 & $0.0945(3.34 \mathrm{~s})$ \\
\hline & & 11 & 99.9765 & $0.0414(12.9 \mathrm{~s})$ & 99.9481 & $0.0945(4.9 \mathrm{~s})$ \\
\hline \multirow{3}{*}{ II } & \multirow{3}{*}{100 (142bp, 62.5s) } & 6 & 100.1008 & $0.0407(4.8 \mathrm{~s})$ & 99.9696 & $0.0940(1.7 \mathrm{~s})$ \\
\hline & & 9 & 99.9384 & $0.0406(9.2 \mathrm{~s})$ & 99.9380 & $0.0942(3.2 \mathrm{~s})$ \\
\hline & & 11 & 100.0655 & $0.0411(12.1 \mathrm{~s})$ & 99.9065 & $0.0945(4.8 \mathrm{~s})$ \\
\hline \multirow{3}{*}{ III } & \multirow{3}{*}{$100(51.75 \mathrm{bp}, 63.0 \mathrm{~s})$} & 6 & 99.9485 & $0.0409(4.5 \mathrm{~s})$ & 99.9461 & $0.1154(3.2 \mathrm{~s})$ \\
\hline & & 9 & 99.9525 & $0.0414(8.6 \mathrm{~s})$ & 99.9430 & $0.1176(7.1 \mathrm{~s})$ \\
\hline & & 11 & 99.9550 & $0.0409(12.7 \mathrm{~s})$ & 99.9275 & $0.1154(9.9 \mathrm{~s})$ \\
\hline \multirow{3}{*}{ IV } & \multirow{3}{*}{$100(62.90 \mathrm{bp}, 64.3 \mathrm{~s})$} & 6 & 100.0927 & $0.0402(5.4 \mathrm{~s})$ & 99.6639 & $0.0953(2.0 \mathrm{~s})$ \\
\hline & & 9 & 99.9982 & $0.0401(9.6 \mathrm{~s})$ & 99.8992 & $0.0984(3.3 \mathrm{~s})$ \\
\hline & & 11 & 100.0421 & $0.0406(15.8 \mathrm{~s})$ & 99.9071 & $0.0953(5.8 \mathrm{~s})$ \\
\hline
\end{tabular}

Table 2. Comparison of numerical values of the GLWB price computed by the finite difference scheme (Forsyth and Vetzal, 2014) and the two regression-based Monte Carlo algorithms.

For all the cases considered, we observe a very good agreement of the numerical values of the GLWB price computed by the two regression-based Monte Carlo algorithms and the finite difference scheme. The numerical results calculated by the LSMC algorithm are slightly higher than the ones calculated by the LB algorithm, illustrating high accuracy of the Monte Carlo algorithms and "biased low" nature of the LB algorithm. The numerical results calculated by the Monte Carlo algorithms using $10^{5}$ simulation paths already achieve sufficiently high precision in a few seconds of CPU time. We notice that the difference in numerical results computed by the LSMC and LB algorithms in Case IV is slightly larger when compared to other cases. The slight decrease in accuracy may be attributed to the no ratchet feature in Case IV. Without the ratchet feature, the simulated values of normalized fund value $\widetilde{W}$ are more scattered and the dispersion of the sample values renders the regressed value less accurate. The increase of the number of basis functions is seen to improve numerical accuracy quite significantly.

We also investigate the separation of the optimal withdrawal strategy regions obtained by the finite difference scheme and the LSMC algorithm at $t=1$. By virtue of homogeneity property, the separation of different regions of optimal withdrawal strategy is characterized by the threshold values of the normalized fund value $W / A$. The numerical values of the threshold values shown in Figure 1 reveal a very good agreement between the finite difference scheme and the LSMC algorithm (using $10^{6}$ simulation paths and 11 Laguerre basis functions with the damping factor $e^{-x / 5}$ ). 


\subsection{Numerical calculations of GLWB price under 3/2-model and ONE model}

The parameter values of the 3/2-model and ONE model used in our numerical calculations are taken from Christoffersen et al. (2010) based on empirical calibration from S\&P 500 option prices. Tables 3 and 4 list the parameter values in the stochastic volatility models and the relevant contractual features in the GLWB product, respectively. In the numerical calculations, we used $10^{6}$ simulation paths, 200 time steps per year in the discretized scheme, the first eleven Laguerre basis functions with $e^{-\frac{x}{5}}$ as the damping factor for the normalized fund value $\tilde{W}$ and the first four Laguerre basis functions with $e^{-\frac{x}{5}}$ as the damping factor for the variance $v$.

\begin{tabular}{cccccccc}
\hline & $\kappa$ & $\theta$ & $\epsilon$ & $v_{0}$ & $\rho$ & $\mu$ & $r$ \\
\hline $3 / 2$ model & 32.8837 & 0.1147 & 7.9115 & 0.1147 & -0.7321 & 0.07 & 0.04 \\
\hline ONE model & 1.7869 & 0.0648 & 1.505 & 0.0648 & -0.7581 & 0.07 & 0.04 \\
\hline
\end{tabular}

Table 3: Parameter values in the 3/2-model and ONE model.

\begin{tabular}{lc}
\hline Parameter & Value \\
\hline Penalty for excess withdrawal, $k(t)$ & $0 \leq t \leq 1: 6 \%, 1<t \leq 2: 5 \%$, \\
& $2 \leq t \leq 3: 4 \%, 3<t \leq 4: 3 \%$, \\
& $4 \leq t \leq 5: 2 \%, 5<t \leq 25: 1 \%$, \\
& $25<t \leq T: 0 \%$ \\
Expiry time, $T$ (years) & 57 \\
Initial payment, $S_{0}$ & 100 \\
Insurance fee, $\eta$ & $1.96 \%$ \\
Mortality & DAV 2004R (65 year old male) \\
& (Pasdika and Wolff, 2005) \\
Mortality payments & At year end \\
Withdrawal rate, $G$ & 0.05 annual \\
Bonus (no withdrawal) & 0.07 annual \\
Ratchet cycle & Every five years \\
Withdrawal strategy & Optimal \\
Withdrawal dates & yearly \\
\hline
\end{tabular}

Table 4: Contract parameters of the GLWB product.

Table 5 reports the numerical values of the GLWB price calculated by the LSMC and LB algorithms under the 3/2-model and ONE model. The difference in the numerical values of the GLWB price obtained from the LSMC and LB algorithms is seen to be small, indicating high accuracy of our algorithms under the stochastic volatility models. The CPU time is higher compared to that of the Geometric Brownian motion shown in Table 2 , due to additional computational effort in the discretized scheme for simulating the stochastic variance and requirement of more basis functions used in the regression. For most practical uses, the speed of computation is sufficiently fast. The fair insurance fees calculated under the $3 / 2$ model and ONE model are very close to each other. Apparently, provided that the model parameters of the stochastic volatility models are well calibrated to market data, the GLWB price is not quite sensitive to the choice of the volatility model. In the following discussion, we may refer to the numerical results in Table 5 as the "base case". 


\begin{tabular}{|c|c|c|c|}
\hline & $J$ & LSMC & LB \\
\hline \multirow{3}{*}{$3 / 2$-model } & 6 & $100.0905(0.0182,686.2 \mathrm{~s})$ & $99.8981(0.0412,752.0 \mathrm{~s})$ \\
& 9 & $100.1560(0.0183,736.1 \mathrm{~s})$ & $99.9335(0.0405,759.2 \mathrm{~s})$ \\
& 11 & $100.0547(0.0182,781.7 \mathrm{~s})$ & $99.8492(0.0407,690.6 \mathrm{~s})$ \\
\hline \multirow{3}{*}{ ONE model } & 6 & $100.1786(0.0166,711.9 \mathrm{~s})$ & $99.9129(0.0414,795.5 \mathrm{~s})$ \\
& 9 & $100.0887(0.0166,723.3 \mathrm{~s})$ & $99.8937(0.0405,798.2 \mathrm{~s})$ \\
& 11 & $100.0832(0.0167,680.9 \mathrm{~s})$ & $99.8742(0.0409,739.5 \mathrm{~s})$ \\
\hline
\end{tabular}

Table 5: Numerical values of the fair price of GLWB that are calculated by the LSMC and LB under the 3/2-model and ONE model. The numerical values shown in brackets are the standard errors and CPU time, respectively. Here, $J$ is the number of basis functions used for the normalized fund value. We use 3 Laguerre basis functions for the variance $v$ in all calculations.

Due to homogeneity property of the GLWB price function, the separation of optimal withdrawal regions is characterized by the normalized fund values $\widetilde{W}$ and stochastic variance $v$. Figure 2 illustrates the separation of the optimal withdrawal regions in the $(\widetilde{W}-v)$ plane on the first withdrawal date for $3 / 2$-model. The choice of the optimal withdrawal strategy is not quite sensitive to the level of stochastic volatility. We do observe that the region of complete surrender decreases when variance $v$ increases. This agrees with the intuition that the embedded option value increases with higher variance. The higher embedded option value lower the propensity of the policyholder to choose "complete surrender".

\subsection{Sensitivity analysis}

We would like to perform sensitivity analysis on the price of the GLWB by varying the model parameters, embedded contractual features and the assumption on the policyholder's withdrawal behavior. We used $10^{5}$ simulation paths and took the physical drift rate $\mu$ to be $7 \%$. In particular, we examine the impact of bonus rate and ratchet provision on the optimal withdrawal strategies and the chance of complete surrender over the policy life.

In Table 6, we show the numerical values of the GLWB price using the LSMC and LB algorithms under the 3/2-model and ONE model with respect to varying bonus rates and cycles of ratchet event. Here, the cycle of ratchet event of $n$ years indicates that the ratchet provision is applied after a time period of $n$ years. Since higher bonus rate and shorter cycle of ratchet event are beneficial to the policyholder, the numerical results verify that the GLWB price increases when the bonus rate is higher and the cycle of ratchet events is shorter. The GLWB price increases by $1.5 \%$ when the cycle of the ratchet events shortens from five years to two years. When we lower the bonus rate from $8 \%$ to $4 \%$, the price decreases by $3 \%$. Interestingly, when the bonus rate further reduces to zero, the price barely decreases when compared to the case of $4 \%$ bonus rate. To explain this result, we observe that when the bonus rate is set at $4 \%$, it still does not provide enough incentive for the policyholder to choose the strategy of $\gamma=0$. The incentive is even lower when the bonus rate becomes lower. These observations are consistent with the numerical studies on optimal withdrawal strategies shown in Figure 3 (to be discussed later). 


\begin{tabular}{cccc}
\hline Bonus rate & Cycle of ratchet event & 3 /2-model & ONE model \\
\hline \multirow{2}{*}{0} & 2 & 100.3315 & 100.1453 \\
& 5 & 98.8762 & 98.8202 \\
\hline \multirow{2}{*}{0.04} & 2 & 100.3270 & 100.1795 \\
& 5 & 98.8981 & 98.8691 \\
\hline \multirow{2}{*}{0.07} & 2 & 101.4455 & 101.4247 \\
& 5 & 99.9888 & 100.0043 \\
\hline \multirow{2}{*}{0.08} & 2 & 103.0922 & 102.9962 \\
& 5 & 101.6105 & 101.6007 \\
\hline
\end{tabular}

Table 6: Sensitivity analysis of the contractual features on the GLWB price under the 3/2-model and ONE model. We list the numerical values of the GLWB price with respect to varying bonus rates and cycles of ratchet (listed in the first two columns). The numerical prices reported are the average of the numerical results calculated by the LSMC and LB algorithms.

In Table 7, we show the numerical values of the GLWB price using both the LSMC and LB algorithms under the 3/2-model and ONE model with respect to varying values of correlation coefficient $\rho$ and volatility of variance $\epsilon$. Similar to the insensitivity to the level of stochastic volatility, the GLWB price is not quite sensitive to the correlation coefficient $\rho$. It is interesting to observe that the price of the GLWB decreases with increasing value of $\epsilon$ under both models. For the 3/2-model, a similar phenomena of price decrease with increasing value of $\epsilon$ has been reported by Yuen and Kwok (2013) in their pricing models of discrete variance swaps.

\begin{tabular}{|c|c|c|c|c|c|c|c|c|c|c|}
\hline \multirow{3}{*}{$3 / 2$-model } & $\epsilon$ & \multicolumn{3}{|c|}{5} & \multicolumn{3}{|c|}{7.9115} & \multicolumn{3}{|c|}{10} \\
\hline & $\rho$ & -0.3 & -0.5 & -0.7 & -0.3 & -0.5 & -0.7 & -0.3 & -0.5 & -0.7 \\
\hline & Price & 103.5930 & 103.5721 & 103.4495 & 100.2829 & 100.1522 & 100.0860 & 98.3864 & 98.3027 & 98.2059 \\
\hline \multirow{3}{*}{ ONE model } & $\epsilon$ & \multicolumn{3}{|c|}{0.5} & \multicolumn{3}{|c|}{1.505} & \multicolumn{3}{|c|}{2.5} \\
\hline & $\rho$ & -0.3 & -0.5 & -0.7 & -0.3 & -0.5 & -0.7 & -0.3 & -0.5 & -0.7 \\
\hline & Price & 100.9381 & 100.8698 & 100.8217 & 100.5581 & 100.3126 & 100.1294 & 99.4801 & 99.2421 & 98.8966 \\
\hline
\end{tabular}

Table 7: Sensitivity analysis of the GLWB price with respect to the model parameters under the 3/2-model and ONE model. We list the numerical values of the GLWB price with varying values of the correlation $\rho$ and the volatility of variance $\epsilon$. The numerical prices reported are the average of the numerical results calculated by the LSMC and LB algorithms.

We would like to examine the percentage of adoption of each of the 3 optimal withdrawal strategies (i) $\gamma=0$, (ii) $\gamma=G A$, (iii) $\gamma=W$ over the policy life under the 3/2-model. The percentage of adoption of one particular optimal withdrawal strategy at time $i$ is calculated as the ratio of the number of simulations paths adopting the strategy at time $i$ over the total number of ongoing paths, with the exclusion of the paths that have been terminated before time $i$ due to complete surrender. The plots in Figure 3 show the probability of each of the 3 optimal withdrawal strategies taken as the optimal choice at time $i$, given that the contract has not been surrendered up to time $i, i=1,2, \ldots, T-1$. Our numerical calculations show that the results under the ONE model are quite similar to the $3 / 2$-model. The plots provide an important information that the writer of the policy can use to estimate the future cashflows. Figure 3 reveals that the zero withdrawal strategy is always suboptimal when the bonus rate is low (at the level of 4\%). When the bonus rate is increased to $7 \%$, the policyholder chooses the zero withdrawal as the optimal strategy on early withdrawal dates with almost 
$100 \%$ certainty. The probability of choosing zero withdrawal becomes lower on later withdrawal dates. After 12 or 13 years since initiation, the zero withdrawal strategy is almost always suboptimal.

Next, we explore the impact of penalty charge on the choice of the optimal strategy of complete surrender. We call the penalty charge setting in Table 4 to be "Penalty 1". In another set of numerical calculations, we consider another penalty charge setting "Penalty 2", the details of which are shown in Table 8. "Penalty 2" has a higher penalty rate than "Penalty 1".

\begin{tabular}{cc}
\hline Penalty for excess withdrawal $\kappa(t)$ & $0 \leq \mathrm{t} \leq 1: 10 \%, 1<t \leq 2: 9 \%$ \\
& $2<\mathrm{t} \leq 3: 8 \%, 3<t \leq 4: 7 \%$ \\
& $4<\mathrm{t} \leq 5: 6 \%, 5<t \leq 25: 5 \%$ \\
& $25<t \leq T: 0 \%$ \\
\hline
\end{tabular}

Table 8: Penalty charge setting "Penalty 2".

We examine the impact of bonus rate and ratchet provision on the distribution of the optimal time for choosing complete surrender over the policy life of the GLWB under the two penalty charge schemes: Penalty 1 (Figure 4) and Penalty 2 (Figure 5). The plots in both figures show that in the fifth year, since there is a ratchet event right after the withdrawal event, the policyholder is prone not to surrender the contract so as to take advantage of the ratchet feature. Hence, the complete surrender strategy tends to be postponed to the next year. While Figure 4 shows that complete surrender may occur in the first few years under Penalty 1 , complete surrender almost does not occur before the sixth year under Penalty 2 (see Figure 5 ). This is because the penalty rate under Penalty 2 is higher, especially at the early life of the contract.

Lastly, we compare the impact of different withdrawal strategies adopted by the policyholder that are commonly assumed in the current literature. The "Suboptimal Strategy 1" means that the policyholder would only take two strategies on each withdrawal date: $\gamma=G A$ and $\gamma=W$. While the strategy of zero withdrawal is always discarded under "Suboptimal Strategy 1", the policyholder would choose the strategy of complete surrender optimally so as to maximize the expected value of the discounted cash flows. The "Suboptimal Strategy 2" refers to the static strategy: the policyholder only takes $\gamma=G A$ until he or she dies. Table 9 shows the significant difference in the GLWB price calculated based on the different assumptions of the policyholder's withdrawal behavior, especially when the bonus rate is high and penalty charge is low. Interestingly, the GLWB prices under "Optimal Strategy" are almost the same as those under "Suboptimal Strategy 1" when the bonus rate is set to be zero or $4 \%$. This is because when the bonus rate is less than or equal to $4 \%$, the zero withdrawal strategy is suboptimal on each withdrawal date with almost $100 \%$ certainty (see Figure 3). 


\begin{tabular}{cccccc}
\hline Bonus rate & Cycle (ratchet event) & Penalty charge & Optimal Strategy & Suboptimal Strategy 1 & Suboptimal Strategy 2 \\
\hline 0 & 2 & 1 & 100.3315 & 100.2746 & 97.8960 \\
0 & 2 & 2 & 98.9781 & 98.9669 & 97.8960 \\
0 & 5 & 1 & 98.8762 & 98.8643 & 95.1451 \\
0 & 5 & 2 & 97.2556 & 97.2013 & 95.1451 \\
0.04 & 2 & 1 & 100.2770 & 100.2746 & 97.8960 \\
0.04 & 2 & 2 & 98.9728 & 98.9669 & 97.8960 \\
0.04 & 5 & 1 & 98.8981 & 98.8643 & 95.1451 \\
0.04 & 5 & 2 & 97.2238 & 97.2013 & 95.1451 \\
0.07 & 2 & 1 & 101.4455 & 100.2746 & 97.8960 \\
0.07 & 2 & 2 & 99.8056 & 98.9669 & 97.8960 \\
0.07 & 5 & 1 & 99.9888 & 98.8643 & 95.1451 \\
0.07 & 5 & 2 & 98.1453 & 97.2013 & 95.1451 \\
0.08 & 2 & 1 & 103.0922 & 100.2746 & 97.8960 \\
0.08 & 2 & 2 & 101.6182 & 98.9669 & 97.8960 \\
0.08 & 5 & 1 & 101.6105 & 98.8643 & 95.1451 \\
0.08 & 5 & 2 & 99.7160 & 97.2013 & 95.1451 \\
\hline
\end{tabular}

Table 9: Comparison of prices of the GLWB product under the optimal withdrawal strategy and two other suboptimal withdrawal strategies, subject to varying values of the bonus rate, cycle of ratchet events and penalty charge schemes.

\section{Conclusion}

We have developed effective regression-based Monte Carlo simulation algorithms for solving the stochastic control models associated with pricing of the Guaranteed Lifelong Withdrawal Benefit (GLWB) in variable annuities. While most earlier pricing models assume the Heston stochastic volatility model for the underlying fund value process, we adopt the 3/2-model and ONE model for the dynamics of the fund value process. We discard the Heston volatility model as a viable stochastic volatility model since empirical studies show that the Heston volatility model leads to inconsistencies in the prescription of volatility dynamics. Thanks to the theoretical result on the preservation of convexity of the European option price function under stochastic volatility models, we derive the bang-bang control strategy on the optimal withdrawal policies. With the simplification of the strategy space of optimal withdrawal policies to only three choices: zero withdrawal, withdrawal at the contractual amount or complete surrender, the solution of the stochastic control GLWB model by the regression-based Monte Carlo simulation algorithm becomes feasible. To enhance computational efficiency, we derive the closed form solution of the GLWB model at zero fund value so that the GLWB price can be obtained by direct numerical evaluation once the fund value in the Monte Carlo simulation path hits zero value. We also establish the convergence proof of the regression-based Monte Carlo simulation algorithms. In the convergence proof, special attentions are paid to deal with the specific features of the stochastic control model and complex jump conditions associated with the bonus and ratchet provisions on the withdrawal dates.

We have performed thorough comparison of the numerical results obtained by the regression-based algorithms (LSMC and LB) and the finite difference scheme under the assumption of the Geometric Brownian motion for the fund value process. Both the LSMC and LB algorithms reveal high level of numerical accuracy and computational efficiency. We have performed extensive sensitivity analysis of the GLWB price function 
with respect to different parameter values in the stochastic control models of GLWB. Similar to other related pricing models under the 3/2-model, the GLWB price function is seen to be a decreasing function in volatility of variance. For both the 3/2-model and ONE model, the GLWB price is not sensitive to varying values of the correlation coefficient between the fund value process and variance process. We also perform the sensitivity analysis of the GLWB function with respect to different contractual features. Our numerical calculations show that high bonus rate and short cycle of ratchet event add more value to the GLWB price, which is consistent with financial intuition. However, when the bonus rate is lower than some threshold, the GLWB price is not sensitive to the bonus rate since the zero withdrawal is always sub-optimal. We also compute the percentage of each optimal withdrawal strategy over the policy life of the GLWB with respect to varying bonus rates and cycles of ratchet event. Our calculations reveal the downward trend in the adoption of the zero withdrawal as the optimal strategy when the policyholder ages. On the other hand, the adoption of the contractual guaranteed withdrawal exhibits an upward trend over the calendar time. Moreover, we study how the cycle of ratchet event and penalty rate influence the optimal time of choosing the strategy of complete surrender. The chance of choosing complete surrender is higher on withdrawal dates that are right after a ratchet event. We also show how the high penalty rate suppresses the propensity of adopting the strategy of complete surrender. In addition, we observe that complete surrender becomes non-optimal as the policyholder ages. This phenomenon is attributed to the higher probability of the policy fund value hitting zero value near the end of contract life. Furthermore, we study the impact of different withdrawal strategy behaviors of the policyholder. The numerical results show that there exists significant difference in the GLWB prices under different assumptions of the policyholder's withdrawal behavior. 


\section{References}

Andreatta, G., Corradin, S., 2003. Valuing the surrender option embedded in a portfolio of Italian life guaranteed participating policies: A least squares Monte Carlo approach. Technical Report of RAS Pianificazione redditività di Gruppo.

Azimzadeh, P., Forsyth, P., 2014. The existence of optimal bang-bang controls for gmxb contracts. Working paper of University of Waterloo.

Bacinello, A.R, Biffis, E., Millossovich, P., 2010. Regression-based algorithms for life insurance contracts with surrender guarantees. Quantitative Finance 10, 1077-1090.

Bacinello, A.R., Millossovich, P. Olivieri, A., Pitacco, E., 2011. Variable annuities: A unifying valuation approach. Insurance: Mathematics and Economics. 49, 285-297.

Baldeaux, J., 2012. Exact simulation of the $3 / 2$ model. International Journal of Theoretical and Applied Finance 15(5), 1-13.

Bauer, D., Kling, A., Russ, J., 2008. A universal pricing framework for guaranteed minimum benefits in variable annuities. ASTIN Bulletin 38, 621-651.

Broadie, M., Kaya, O., 2006. Exact simulation of stochastic volatility and other affine jump diffusion processes. Operations Research 54(2), 217-231.

Chen, Z., Vetzal, K., Forsyth, P., 2008. The effect of modelling parameters on the value of GMWB guarantees. Insurance: Mathematics and Economics 43, 165-173.

Christoffersen, P., Jacobs, K., Mimouni, K., 2010. Volatility dynamics for the S\&P500: evidence from realized volatility, daily returns, and option prices. Review of Financial Studies 23(8), 3141-3189.

Clément, E., Lamberton, D., Protter, P., 2002. An analysis of a least squares regression method for American option pricing. Finance and Stochastics 6(4), 449-471.

Dai, M., Kwok, Y.K., Zong, J., 2008. Guaranteed minimum withdrawal benefit in variable annuities. Mathematical Finance 18, 595-611.

Donelly, R., Jaimungal, S., Rubisov, D.H., 2014. Valuing guaranteed withdrawal benefits with stochastic interest rates and volatility. Quantitative Finance 14, 369-382.

Forsyth, P., Vetzal, K., 2014. An optimal stochastic control framework for determining the cost of hedging of variable annuities. Journal of Economic Dynamics and Control 44, 29-53.

Glasserman, P., 2004. Monte Carlo methods in financial engineering. Springer.

Heston, S.L., 1993. A closed-form solution for options with stochastic volatility with applications to bond and currency options. Review of Financial Studies 6(2), 327-343.

Higham, D.J., Mao, X., 2005. Convergence of the Monte Carlo simulations involving the mean reverting square root process. Journal of Computational Finance 8(3), 35-62.

Hobson, D., 2010. Comparison results for stochastic volatility models via coupling. Finance and Stochastics $14(1), 129-152$. 
Holz, D., Kling, A., Russ, J., 2012. GMWB for life: An analysis of lifelong withdrawal guarantees. Zeitschrift fur die gesamte Versicherungswissenschaft 101, 305-325.

Huang, H., Milevsky, M.A., Salisbury, T.S., 2014. Optimal initiation of a GLWB in a variable annuity: No Arbitrage approach. Insurance: Mathematics and Economics 56, 102-111.

Huang, Y., Forsyth, P.A., 2012. Analysis of a penalty method for pricing a guaranteed minimum withdrawal benefit (GMWB). IMA Journal of Numerical Analysis 32(1), 320-351.

Huang, Y.T., Kwok, Y.K. 2014. Analysis of optimal dynamic withdrawal policies in withdrawal guarantee products. To appear in Journal of Economic Dynamics and Control.

Ishida, I., Engle, R., 2002. Modeling variance of variance: The square root, the affine and the CEV GARCH models. Working Paper of New York University.

Javaheri, A., 2004. The volatility process: A study of stock market dynamics via parametric stochastic volatility models and a comparison to the information embedded in option prices. PhD dissertation, Ecole des Mines de Paris.

Kling, A., Ruez, F., Ruß, J., 2013. The impact of policyholder behavior on pricing, hedging, and hedge efficiency of withdrawal benefit guarantees in variable annuities. Working paper of Ulm University.

Longstaff, F.A., Schwartz, E.S., 2001. Valuing American options by simulation: A simple least-squares approach. Review of Financial Studies 14(1), 113-147.

Lord, R. Koekkoek, R., Van Dijk, D., 2010. A comparison of biased simulation schemes for stochastic volatility models. Quantitative Finance 10(2), 177-194.

Milevsky, M.A., Salisbury, T.S., 2006. Financial valuation of guaranteed minimum withdrawal benefits. Insurance: Mathematics and Economics 38(1), 21-38.

Pasdika, U., Wolff, J., 2005. Coping with longevity: The new German annuity valuation table DAV $2004 \mathrm{R}$. The Living to 100 and Beyond Symposium, Orlando Florida.

Peng, J., Leung, K.S., Kwok, Y.K., 2012. Pricing guaranteed minimum withdrawal benefits under stochastic interest rates. Quantitative Finance 12, 933-941.

Piscopo, G., Haberman, S., 2011. The valuation of guaranteed lifelong withdrawal benefit options in variable annuity contracts and the impact of mortality risk. North American Actuarial Journal 15(1), 59-76.

Shah, P., Bertsimas, D., 2008. An analysis of the guaranteed withdrawal benefits for life option. Working paper of Sloan School of Management, MIT.

Steinorth, P., Mitchell, O.S., 2012. Valuing variable annuities with guaranteed minimum lifetime withdrawal benefits. Working paper of St John's University and University of Pennsylvania.

Tsitsiklis, J. N., Van Roy, B., 2001. Regression methods for pricing complex American style options. Neural Networks, IEEE Transactions 12(4), 694-703.

Yuen, C.H., Kwok, Y.K., 2013. Pricing exotic variance swaps under 3/2-stochastic volatility models. Working paper of Hong Kong University of Science and Technology. 


\section{Appendix A - Proof of Proposition 1}

It is convenient to express the governing stochastic differential equations for the $3 / 2$-model in terms of $W_{t}$ and $u_{t}=\sqrt{v_{t}}$ under the filtered probability space $(\Omega, \mathcal{F}, Q)$ as follows

$$
\begin{aligned}
d W_{t} & =(r-\eta) W_{t} d t+u_{t} W_{t} d B_{t}^{W} \\
d u_{t} & =\left(a u_{t}+b u_{t}^{3}\right) d t+c u_{t}^{2} d B_{t}^{u}
\end{aligned}
$$

with $d B_{t}^{W} d B_{t}^{u}=\rho d t, a=\frac{\kappa \theta}{2}, b=-\frac{\kappa}{2}-\frac{\epsilon^{2}}{8}$ and $c=\frac{\epsilon}{2}$. According to Theorem 3.1 in Hobson (2010), the corresponding coupling of the pair $\left(W_{t}, u_{t}\right)$ is given by the following pair $\left(X_{t}, Y_{t}\right)$ that satisfy

$$
\begin{aligned}
d X_{t} & =X_{t} d B_{t}^{X} \\
d Y_{t} & =\left(\frac{a}{Y_{t}}+b Y_{t}\right) d t+c Y_{t} d B_{t}^{Y},
\end{aligned}
$$

with $d B_{t}^{X} d B_{t}^{Y}=\rho d t, X_{0}=W_{0}$ and $Y_{0}=u_{0}$. Based on Proposition 6.1 in Hobson (2010), the convexity preservation property of the European price function is observed in the pair $\left(W_{t}, u_{t}\right)$ provided that there exists a strong solution to the pair $\left(X_{t}, Y_{t}\right)$ defined by eqs. (A.2a,b). The proof of Proposition 1 is completed if we manage to derive a strong solution for eqs. (A.2a,b).

First, we would like to establish the following technical result. Let $M_{t}$ be governed by the stochastic differential equation of the form

$$
d M_{t}=f\left(M_{t}, t\right) d t+g(t) M_{t} d B_{t}, \quad M_{0}=m
$$

where $f$ and $g$ are continuous (deterministic) functions. Let the integrating factor $F_{t}$ be defined by

$$
F_{t}=\exp \left(-\int_{0}^{t} g(s) d B_{s}+\int_{0}^{t} \frac{g^{2}(s)}{2} d s\right) .
$$

We then have

$$
d\left(F_{t} M_{t}\right)=F_{t} f\left(M_{t}, t\right) d t
$$

The proof of this technical result can be established by direct calculus procedure. Next, we derive the (strong) solution of eq.(A.2b) by applying eq. (A.3). Let $f(y, t)=\frac{a}{y}+b y$ and $g(t)=c$, the corresponding integrating factor $F_{t}$ is found to be

$$
F_{t}=e^{\frac{1}{2} c^{2} t-c B_{t}^{Y}}
$$

Define $Z_{t}=F_{t} Y_{t}$ and by formula (A.3), we obtain

$$
d Z_{t}=F_{t} f\left(\frac{Z_{t}}{F_{t}}, t\right) d t=\left(b Z_{t}+\frac{a F_{t}^{2}}{Z_{t}}\right) d t .
$$


Let $H_{t}=e^{-b t} Z_{t}$, we then have

$$
d H_{t}^{2}=2 a e^{-2 b t} F_{t}^{2} d t
$$

Integrating the equation and taking the positive square root, we obtain the following strong solution for $Y_{t}$

$$
Y_{t}=\frac{Z_{t}}{F_{t}}=e^{c B_{t}^{Y}+\left(b-\frac{1}{2} c^{2}\right) t}\left[\int_{0}^{t} 2 a e^{\left(c^{2}-2 b\right) s-2 c B_{s}^{Y}} d s+v^{2}\right]^{\frac{1}{2}} \text {. }
$$

It is obvious that there exists a strong solution for eq. (A.2a) since the coefficient satisfies the Lipschitz condition. By Proposition 6.1 in Hobson (2010), we then conclude that the 3/2-model observes the preservation of convexity in the European price function.

\section{Appendix B - Proof of Proposition 2}

We would like to establish that once it is optimal to withdraw the contractual amount $G A$ on some withdrawal date, then it would be non-optimal to choose zero withdrawal on all subsequent withdrawal dates. For simplicity of the proof, we may assume the bonus rate $b_{i}$ to be the same for all $i$. The result remains valid since the decreasing bonus rate gives more incentive for earlier adoption of $\gamma=0$ strategy. It suffices to show that the following pattern of sequence of withdrawal policies with withdrawal dates of adopting $\gamma=0$ strategy lying between withdrawal dates of adopting $\gamma=G A$

$$
\begin{array}{ccccc}
\text { GA } & 0 & \cdots & 0 & \text { GA } \\
j^{\text {th }} \text { date } & & & & k^{\text {th }} \text { date }
\end{array}
$$

cannot be optimal. We prove by contradiction. Suppose we swap the strategy $\gamma=0$ on the $(j+1)^{t h}$ date with the strategy $\gamma=G A$ on the $k^{\text {th }}$ date to obtain the following sequence

$$
\begin{array}{ccccc}
\text { GA } & \text { GA } & 0 & \cdots & 0 \\
j^{\text {th }} \text { date } & (j+1)^{\text {th }} \text { date } & & & k^{\text {th }} \text { date }
\end{array}
$$

Let $b$ denote the (constant) bonus rate. Since the first sequence of strategies is optimal, so we have

$$
e^{-r(k-t)} p_{k}(1+b)^{k-j-1} G A_{j+1}>e^{-r(j+1-t)} p_{j+1} G A_{j+1}
$$

Simplifying ineq. (B.1), we obtain

$$
e^{-r}(1+b)>\left(\frac{p_{j+1}}{p_{k}}\right)^{\frac{1}{k-j-1}} .
$$

Alternatively, we consider the third sequence of strategies which is obtained by swapping the strategy $\gamma=0$ on the $(j+1)^{t h}$ date by the strategy $\gamma=G A$ on the $j^{\text {th }}$ date, where

$$
\begin{array}{ccccc}
0 & \mathrm{GA} & 0 & \cdots & \mathrm{GA} \\
j^{\text {th }} \text { date } & (j+1)^{\text {th }} \text { date } & & & k^{\text {th }} \text { date }
\end{array}
$$

Again, since the first sequence of withdrawal strategies is optimal, so we deduce that

$$
e^{-r j} p_{j} G A_{j}>e^{-r(j+1)} p_{j+1}(1+b) G A_{j}
$$


Combining with ineq. (B.2) and ineq. (B.3) yields

$$
\frac{p_{j}}{p_{j+1}}>e^{-r}(1+b)>\left(\frac{p_{j+1}}{p_{k}}\right)^{\frac{1}{k-j-1}} \geq \frac{p_{j+1}}{p_{j+2}}
$$

where last inequality is deduced by

$$
\begin{aligned}
\left(\frac{p_{j+1}}{p_{k}}\right)^{\frac{1}{k-j-1}} & =\left(\frac{p_{j+1}}{p_{j+2}} \frac{p_{j+2}}{p_{j+3}} \ldots \frac{p_{k-1}}{p_{k}}\right)^{\frac{1}{k-j-1}} \\
& =\left(\frac{1}{1-q_{j+1}} \frac{1}{1-q_{j+2}} \cdots \frac{1}{1-q_{k-1}}\right)^{\frac{1}{k-j-1}} \quad\left(\text { since } q_{j+1} \leq q_{j+2} \leq \cdots \leq q_{k-1}\right) \\
& \geq \frac{1}{1-q_{j+1}} \\
& =\frac{p_{j+1}}{p_{j+2}} .
\end{aligned}
$$

The derived result of $\frac{p_{j}}{p_{j+1}}>\frac{p_{j+1}}{p_{j+2}}$ in ineq. (B.4) is contradicting with the assumption of $q_{j} \leq q_{j+1}$. Hence, once it is optimal to withdraw the contractual amount on some date $i_{G}^{*}$, the optimal withdrawal strategy is to continue to withdraw the contractual amount on all subsequent withdrawal dates.

Once we have established the above optimal withdrawal policy on all withdrawal dates when the fund value is zero, the solution of $V_{0}(A, t)$ amounts to the determination of $i_{G}^{*}$ such that the following sum of discounted expected withdrawal amounts

$$
\prod_{i=i_{0}}^{i_{G}^{*}-1}\left(1+b_{i}\right)\left[\sum_{k=i_{G}^{*}}^{T-1} e^{-r(k-t)} p_{k}\right] G A
$$

is maximized. Hence, $V_{0}(A, t)$ is given by eq. (3.3).

\section{Appendix C - Proof of Proposition 3}

We proceed by induction on $i$. It is clear that the result is true for $i=T$ since both value functions are equal to the terminal payoff. Suppose it holds for $i+1$, we want to show that it is also true for $i$. Note that $V\left(W, A, v, i^{-}\right)$and $V\left(W, A, v, i^{-} ; \boldsymbol{\beta}_{i}^{(J)}\right)$ are given by

$$
\begin{aligned}
V\left(W, A, v, i^{-}\right) & =p_{i-1} q_{i-1} W_{i^{-}}+p_{i} \max \left\{f_{i}\left(W_{i^{-}} ; A_{i^{-}}\right)\right. \\
& e^{-r} h_{2}\left(W_{i^{-}}, A_{i^{-}} ; 0\right) E\left[\widetilde{V}\left(\widetilde{\mathbf{X}},(i+1)^{-}\right) \mid \widetilde{\mathbf{X}} i=\left(\widetilde{h}_{1}\left(W_{i^{-}}, A_{i^{-}} ; 0\right), v\right)\right] \\
& \left.G A_{i^{-}}+e^{-r} h_{2}\left(W_{i^{-}}, A_{i^{-}} ; G A_{i^{-}}\right) E\left[\widetilde{V}\left(\widetilde{\mathbf{X}},(i+1)^{-}\right) \mid \widetilde{\mathbf{X}}_{i}=\left(\widetilde{h}_{1}\left(W_{i^{-}}, A_{i^{-}} ; G A_{i^{-}}\right), v\right)\right]\right\}
\end{aligned}
$$


and

$$
\begin{aligned}
V\left(W, A, v, i^{-} ; \boldsymbol{\beta}_{i}^{(J)}\right) & =p_{i-1} q_{i-1} W_{i^{-}}+p_{i} \max \left\{f_{i}\left(W_{i^{-}} ; A_{i^{-}}\right)\right. \\
& e^{-r} h_{2}\left(W_{i^{-}}, A_{i^{-}} ; 0\right) \Psi^{(J)}\left(\tilde{h}_{1}\left(W_{i^{-}}, A_{i^{-}} ; 0\right), v\right) \boldsymbol{\beta}_{i}^{(J)} \\
& \left.G A_{i^{-}}+e^{-r} h_{2}\left(W_{i^{-}}, A_{i^{-}} ; G A_{i^{-}}\right) \Psi^{(J)}\left(\tilde{h}_{1}\left(W_{i^{-}}, A_{i^{-}} ; G A_{i^{-}}\right), v\right) \boldsymbol{\beta}_{i}^{(J)}\right\} .
\end{aligned}
$$

We would like to remark on the notational interpretation of the two value functions: $V\left(W, A, v, i^{-}\right)$and $V\left(W, A, v, i^{-} ; \boldsymbol{\beta}_{i}^{(J)}\right)$. The conditional expectation is approximated by the regressed value based on the regression coefficients $\boldsymbol{\beta}_{i}^{(J)}$ in the determination of $V\left(W, A, v, i^{-} ; \boldsymbol{\beta}_{i}^{(J)}\right)$, whereas $V\left(W, A, v, i^{-}\right)$represents the exact value function without using any approximation on the conditional expectation. The absolute difference $\left|V\left(W, A, v, i^{-} ; \boldsymbol{\beta}_{i}^{(J)}\right)-V\left(W, A, v, i^{-}\right)\right|$depends on the optimal strategy $\gamma_{i}^{*(J)}$ and $\gamma_{i}^{*}$. There are nine combinations of the pair of optimal strategies as shown in the table below. For illustration, we only show the convergence of the absolute difference to zero as $J$ tends to infinity for case 2 . The convergence of the absolute difference to zero for all other cases can be established in a similar manner.

\begin{tabular}{|c|c|c|c|}
\hline$\gamma^{*} \gamma^{*(J)}$ & 0 & $G A$ & $W$ \\
\hline 0 & 1 & 2 & 3 \\
\hline$G A$ & 4 & 5 & 6 \\
\hline$W$ & 7 & 8 & 9 \\
\hline
\end{tabular}

For case 2, it is slightly more complicated than case 5 due to discrepancy in the optimal strategies. We rewrite the absolute difference of the two value functions as follows

$$
\begin{aligned}
& \left|V\left(W, A, v, i^{-} ; \boldsymbol{\beta}_{i}^{(J)}\right)-V\left(W, A, v, i^{-}\right)\right| \\
= & {\left[V\left(W, A, v, i^{-} ; \boldsymbol{\beta}_{i}^{(J)}\right)-V\left(W, A, v, i^{-}\right)\right] \mathbf{1}_{\left\{V\left(W, A, v, i^{-} ; \boldsymbol{\beta}_{i}^{(J)}\right) \geq V\left(W, A, v, i^{-}\right)\right\}} } \\
& +\left[V\left(W, A, v, i^{-}\right)-V\left(W, A, v, i^{-} ; \boldsymbol{\beta}_{i}^{(J)}\right)\right] \mathbf{1}_{\left\{V\left(W, A, v, i^{-} ; \boldsymbol{\beta}_{i}^{(J)}\right)<V\left(W, A, v, i^{-}\right)\right.} .
\end{aligned}
$$

Since the optimal strategies under case 2 for $V\left(W, A, v, i^{-} ; \boldsymbol{\beta}_{i}^{(J)}\right)$ and $V\left(W, A, v, i^{-}\right)$are assumed to be $G A$ and 0 , respectively, we bound the first term in (C.2) by shifting to the sub-optimal strategy of $G A$ for $V\left(W, A, v, i^{-}\right)$. 
We then have

$$
\begin{aligned}
& {\left[V\left(W, A, v, i^{-} ; \boldsymbol{\beta}_{i}^{(J)}\right)-V\left(W, A, v, i^{-}\right)\right] \mathbf{1}_{\left\{V\left(W, A, v, i^{-} ; \boldsymbol{\beta}_{i}^{(J)}\right) \geq V\left(W, A, v, i^{-}\right)\right\}} } \\
= & p_{i} e^{-r}\left\{G A_{i^{-}}+h_{2}\left(W_{i^{-}}, A_{i^{-}} ; G A_{i^{-}}\right) \Psi^{(J)}\left(\widetilde{h}_{1}\left(W_{i^{-}}, A_{i^{-}} ; G A_{i^{-}}\right), v\right) \boldsymbol{\beta}_{i}^{(J)}\right. \\
& \left.\quad-h_{2}\left(W_{i^{-}}, A_{i^{-}} ; 0\right) E\left[\widetilde{V}\left(\widetilde{\mathbf{X}},(i+1)^{-}\right) \mid \widetilde{\mathbf{X}}_{i}=\left(\widetilde{h}_{1}\left(W_{t_{i, d}^{-}}, A_{t_{i, d}^{-}}, 0\right), v\right)\right]\right\} \mathbf{1}_{\left\{V\left(W, A, v, i^{-} ; \boldsymbol{\beta}_{i}^{(J)}\right) \geq V\left(W, A, v, i^{-}\right)\right\}} \\
\leq & p_{i} e^{-r}\left\{G A_{i^{-}}+h_{2}\left(W_{i^{-}}, A_{i^{-}} ; G A_{i^{-}}\right) \Psi^{(J)}\left(\widetilde{h}_{1}\left(W_{i^{-}}, A_{i^{-}} ; G A_{i^{-}}\right), v\right) \boldsymbol{\beta}_{i}^{(J)}-G A_{i^{-}}\right. \\
& \left.\left.\quad-h_{2}\left(W_{i^{-}}, A_{i^{-}} ; G A_{i^{-}}\right) E\left[\widetilde{V}\left(\widetilde{\mathbf{X}},(i+1)^{-}\right) \mid \widetilde{\mathbf{X}}_{i}=\left(\widetilde{h}_{1}\left(W_{t_{i, d}^{-}}, A_{t_{i, d}^{-}}, G A_{i^{-}}\right), v\right)\right]\right\} \mathbf{1}_{\left\{V\left(W, A, v, i^{-} ; \boldsymbol{\beta}_{i}^{(J)}\right) \geq V\left(W, A, v, i^{-}\right)\right\}}\right) \\
= & p_{i} e^{-r} h_{2}\left(W_{i^{-}}, A_{i^{-}} ; G A_{i^{-}}\right)\left\{\Psi^{(J)}\left(\widetilde{h}{ }_{1}\left(W_{i^{-}}, A_{i^{-}} ; G A_{i^{-}}\right), v\right) \boldsymbol{\beta}_{i}^{(J)}\right. \\
& \left.-E\left[\widetilde{V}\left(\widetilde{\mathbf{X}},(i+1)^{-}\right) \mid \widetilde{\mathbf{X}}{ }_{i}=\left(\widetilde{h}_{1}\left(W_{i^{-}}, A_{i^{-}} ; G A_{i^{-}}\right), v\right)\right]\right\} \mathbf{1}_{\left\{V\left(W, A, v, i^{-} ; \boldsymbol{\beta}_{i}^{(J)}\right) \geq V\left(W, A, v, i^{-}\right)\right\}} .
\end{aligned}
$$

Let $\operatorname{Proj}_{i}^{(J)}$ be the orthogonal projection onto the span of $\psi_{1}, \ldots, \psi_{J}$ with respect to the state variable $\widetilde{X}_{i}$ and $L^{2}$ norm. Note that

$$
\begin{aligned}
& \Psi^{(J)}\left(\widetilde{h}_{1}\left(W_{i^{-}}, A_{i^{-}} ; G A_{i^{-}}\right), v\right) \boldsymbol{\beta}_{i}^{(J)} \\
= & \operatorname{Proj}_{i}^{(J)}\left(E\left[\widetilde{V}\left(\widetilde{\mathbf{X}},(i+1)^{-} ; \boldsymbol{\beta}_{i+1}^{(J)}\right) \mid \widetilde{\mathbf{X}}_{i}=\left(\widetilde{h}_{1}\left(W_{i^{-}}, A_{i^{-}}, G A_{i^{-}}\right), v\right)\right]\right) \\
= & \operatorname{Proj}_{i}^{(J)}\left(E\left[\widetilde{V}\left(\widetilde{\mathbf{X}},(i+1)^{-} ; \boldsymbol{\beta}_{i+1}^{(J)}\right)-\widetilde{V}\left(\widetilde{\mathbf{X}},(i+1)^{-}\right) \mid \widetilde{\mathbf{X}}_{i}=\left(\widetilde{h}_{1}\left(W_{i^{-}}, A_{i^{-}}, G A_{i^{-}}\right), v\right)\right]\right) \\
& \quad \operatorname{Proj}_{i}^{(J)}\left(E\left[\widetilde{V}\left(\widetilde{\mathbf{X}},(i+1)^{-}\right) \mid \widetilde{\mathbf{X}}{ }_{i}=\left(\widetilde{h}_{1}\left(W_{i^{-}}, A_{i^{-}}, G A_{i^{-}}\right), v\right)\right]\right) .
\end{aligned}
$$

Consequently, we obtain

$$
\begin{aligned}
& \quad\left[V\left(W, A, v, i^{-} ; \boldsymbol{\beta}_{i}^{(J)}\right)-V\left(W, A, v, i^{-}\right)\right] \mathbf{1}_{\left\{V\left(W, A, v, i^{-} ; \boldsymbol{\beta}_{i}^{(J)}\right) \geq V\left(W, A, v, i^{-}\right)\right\}} \\
& \leq p_{i} e^{-r}\left|h_{2}\left(W_{i^{-}}, A_{i^{-}} ; G A_{i^{-}}\right)\right|\left\{\left|\operatorname{Proj}_{i}^{(J)}\left(E\left[\widetilde{V}\left(\widetilde{\mathbf{X}},(i+1)^{-} ; \boldsymbol{\beta}_{i+1}^{(J)}\right)-\widetilde{V}\left(\widetilde{\mathbf{X}},(i+1)^{-}\right) \mid \widetilde{\mathbf{X}}_{i}=\left(\widetilde{h}_{1}\left(W_{i^{-}}, A_{i^{-}}, G A_{i^{-}}\right), v\right)\right]\right)\right|\right. \\
& \quad+\mid \operatorname{Proj}_{i}^{(J)}\left(E\left[\widetilde{V}\left(\widetilde{\mathbf{X}},(i+1)^{-}\right) \mid \widetilde{\mathbf{X}}_{i}=\left(\widetilde{h}_{1}\left(W_{i^{-}}, A_{i^{-}}, G A_{i^{-}}\right), v\right)\right]\right) \\
& \left.\quad-E\left[\widetilde{V}\left(\widetilde{\mathbf{X}},(i+1)^{-}\right) \mid \widetilde{\mathbf{X}}_{i}=\left(\widetilde{h}_{1}\left(W_{i^{-}}, A_{i^{-}}, G A_{i^{-}}\right), v\right)\right] \mid\right\} \mathbf{1}_{\left\{V\left(W, A, v, i^{-} ; \boldsymbol{\beta}_{i}^{(J)}\right) \geq V\left(W, A, v, i^{-}\right)\right\}} .
\end{aligned}
$$


Based on the property of orthogonal projection $\operatorname{Proj}_{i}^{(J)}$, we obtain

$$
\begin{aligned}
& \|\|\left[V\left(W, A, v, i^{-} ; \boldsymbol{\beta}_{i}^{(J)}\right)-V\left(W, A, v, i^{-}\right)\right] \mathbf{1}_{\left\{V\left(W, A, v, i^{-} ; \boldsymbol{\beta}_{i}^{(J)}\right) \geq V\left(W, A, v, i^{-}\right)\right\}} \|_{2} \\
& \leq\left\|p_{i} e^{-r} h_{2}\left(W_{i^{-}}, A_{i^{-}} ; G A_{i^{-}}\right)\right\|_{2}\left\{\left\|\operatorname{Proj}_{i}^{(J)}\left(E\left[\widetilde{V}\left(\widetilde{\mathbf{X}},(i+1)^{-} ; \boldsymbol{\beta}_{i+1}^{(J)}\right)-\widetilde{V}\left(\widetilde{\mathbf{X}},(i+1)^{-}\right) \mid \widetilde{\mathbf{X}}_{i}=\left(\widetilde{h}_{1}\left(W_{i^{-}}, A_{i^{-}}, G A_{i^{-}}\right), v\right)\right]\right)\right\|_{2}\right. \\
& \quad+\| \operatorname{Proj}_{i}^{(J)}\left(E\left[\widetilde{V}\left(\widetilde{\mathbf{X}},(i+1)^{-}\right) \mid \widetilde{\mathbf{X}}_{i}=\left(\widetilde{h}_{1}\left(W_{i^{-}}, A_{i^{-}}, G A_{i^{-}}\right), v\right)\right]\right) \\
& \left.\quad-E\left[\widetilde{V}\left(\widetilde{\mathbf{X}},(i+1)^{-}\right) \mid \widetilde{\mathbf{X}}_{i}=\left(\widetilde{h}_{1}\left(W_{i^{-}}, A_{i^{-}}, G A_{i^{-}}\right), v\right)\right] \|_{2}\right\} \mathbf{1}_{\left\{V\left(W, A, v, i^{-} ; \boldsymbol{\beta}_{i}^{(J)}\right) \geq V\left(W, A, v, i^{-}\right)\right\}} \cdot \\
& \leq\left\|p_{i} e^{-r} h_{2}\left(W_{i^{-}}, A_{i-} ; G A_{i^{-}}\right)\right\|_{2}\left\{\left\|\widetilde{V}\left(\widetilde{\mathbf{X}},(i+1)^{-} ; \boldsymbol{\beta}_{i+1}^{(J)}\right)-\widetilde{V}\left(\widetilde{\mathbf{X}},(i+1)^{-}\right)\right\|_{2}\right. \\
& \quad+\| \operatorname{Proj}_{i}^{(J)}\left(E\left[\widetilde{V}\left(\widetilde{\mathbf{X}},(i+1)^{-}\right) \mid \widetilde{\mathbf{X}} i_{i}=\left(\widetilde{h}_{1}\left(W_{i^{-}}, A_{i^{-}}, G A_{i^{-}}\right), v\right)\right]\right) \\
& \left.\quad-E\left[\widetilde{V}\left(\widetilde{\mathbf{X}},(i+1)^{-}\right) \mid \widetilde{\mathbf{X}}{ }_{i}=\left(\widetilde{h}_{1}\left(W_{i^{-}}, A_{i^{-}}, G A_{i^{-}}\right), v\right)\right] \|_{2}\right\} .
\end{aligned}
$$

The first term inside the curl bracket in (C.3) tends to zero as $J$ goes to infinity due to the inductive assumption. The second term vanishes as $J$ goes to infinity due to H.1 condition. Similarly, one can show the second term in (C.2) tends to zero with respect to $L^{2}$ norm as $J$ goes to infinity. Since $\left\|V\left(W, A, v, i^{-} ; \beta_{i}^{(J)}\right)-V\left(W, A, v, i^{-}\right)\right\|_{2}$ is equal to the summation of $L^{2}$ norm of the two terms in (C.2), the result in eq. (3.13) is established.

\section{Appendix D - Proof of Lemma 4}

We proceed by induction on $i$. For $i=T-1$, we have

$$
\boldsymbol{\beta}_{T-1}^{(M, J)}=\left(R_{T-1}^{(M, J)}\right)^{-1} \frac{1}{M} \sum_{m=1}^{M} p_{T-1} \widetilde{W}_{T}^{(m)} \Psi^{(J)}\left(\widetilde{\mathbf{X}}_{T-1}^{(m)}\right)
$$

Here, $R_{T-1}^{(M, J)}$ is an $J \times J$ matrix with coefficients given by

$$
\left(R_{T-1}^{(M, J)}\right)_{1 \leq k, l \leq J}=\frac{1}{M} \sum_{m=1}^{M} \psi_{k}\left(\widetilde{\mathbf{X}}_{T-1}^{(m)}\right) \psi_{l}\left(\widetilde{\mathbf{X}}_{T-1}^{(m)}\right) .
$$

By the law of large numbers, we obtain

$$
\begin{aligned}
\boldsymbol{\beta}_{T-1}^{(M, J)} & =\left(R_{T-1}^{(M, J)}\right)^{-1} \frac{1}{M} \sum_{m=1}^{M} p_{T-1} \widetilde{W}_{T}^{(m)} \Psi^{(J)}\left(\widetilde{\mathbf{X}}_{T-1}^{(m)}\right) \\
& \rightarrow\left(R_{T-1}^{(J)}\right)^{-1} E\left[p_{T-1} \widetilde{W}_{T} \Psi^{(J)}\left(\widetilde{\mathbf{X}}_{T-1}\right)\right] \quad \text { a.s., as } M \text { goes to infinity } \\
& =\boldsymbol{\beta}_{T-1}^{(J)} .
\end{aligned}
$$


Now, suppose the result is true for $i+1$, we would like to show the result is also true for $i$. We express $\boldsymbol{\beta}_{i}^{(M, J)}$ as follows

$$
\boldsymbol{\beta}_{i}^{(M, J)}=\left(R_{i}^{(M, J)}\right)^{-1} \frac{1}{M} \sum_{m=1}^{M} \widetilde{V}\left(\widetilde{\mathbf{X}},(i+1)^{-} ; \boldsymbol{\beta}_{i+1}^{(M, J)}\right) \Psi^{(J)}\left(\widetilde{\mathbf{X}}_{i}^{(m)}\right) .
$$

By the law of large numbers, we know that $R_{i}^{(M, J)}$ converges almost surely to $R_{i}^{(J)}$. To claim that $\boldsymbol{\beta}_{i}^{(M, J)}$ converges to $\boldsymbol{\beta}_{i}^{(J)}$, it suffices to show

$$
\begin{aligned}
& \frac{1}{M} \sum_{m=1}^{M}\left[\widetilde{V}\left(\widetilde{\mathbf{X}}^{(m)},(i+1)^{-} ; \boldsymbol{\beta}_{i+1}^{(M, J)}\right) \Psi^{(J)}\left(\widetilde{\mathbf{X}}_{i}^{(m)}\right)\right. \\
& \left.\quad-\widetilde{V}\left(\widetilde{\mathbf{X}}^{(m)},(i+1)^{-} ; \boldsymbol{\beta}_{i+1}^{(J)}\right) \Psi^{(J)}\left(\widetilde{\mathbf{X}}_{i}^{(m)}\right)\right] \rightarrow 0 \text { a.s., as } M \rightarrow \infty .
\end{aligned}
$$

The Euclidean norm of above difference is bounded by

$$
\begin{aligned}
& \frac{1}{M} \sum_{m=1}^{M}\left|\Psi^{(J)}\left(\widetilde{\mathbf{X}}_{i}^{(m)}\right)\right|\left|\widetilde{V}\left(\widetilde{\mathbf{X}}^{(m)},(i+1)^{-} ; \boldsymbol{\beta}_{i+1}^{(M, J)}\right)-\widetilde{V}\left(\widetilde{\mathbf{X}}^{(m)},(i+1)^{-} ; \boldsymbol{\beta}_{i+1}^{(J)}\right)\right| \\
& =\frac{1}{M} \sum_{m=1}^{M}\left|\Psi^{(J)}\left(\widetilde{\mathbf{X}}_{i}^{(m)}\right)\right|\left\{\left[\widetilde{V}\left(\widetilde{\mathbf{X}}^{(m)},(i+1)^{-} ; \boldsymbol{\beta}_{i+1}^{(M, J)}\right)-\widetilde{V}\left(\widetilde{\mathbf{X}}^{(m)},(i+1)^{-} ; \boldsymbol{\beta}_{i+1}^{(J)}\right)\right]\right. \\
& \mathbf{1}_{\left\{\widetilde{V}\left(\widetilde{\mathbf{X}}^{(m)},(i+1)^{-} ; \boldsymbol{\beta}_{i+1}^{(M, J)}\right) \geq \widetilde{V}\left(\widetilde{\mathbf{X}}^{(m)},(i+1)^{-} ; \boldsymbol{\beta}_{i+1}^{(J)}\right)\right\}}+\left[\widetilde{V}\left(\widetilde{\mathbf{X}}^{(m)},(i+1)^{-} ; \boldsymbol{\beta}_{i+1}^{(J)}\right)-\widetilde{V}\left(\widetilde{\mathbf{X}}^{(m)},(i+1)^{-} ; \boldsymbol{\beta}_{i+1}^{(M, J)}\right)\right] \\
& \left.\mathbf{1}_{\left\{\widetilde{V}\left(\widetilde{\mathbf{X}}^{(m)},(i+1)^{-} ; \boldsymbol{\beta}_{i+1}^{(M, J)}\right)<\widetilde{V}\left(\widetilde{\mathbf{X}}^{(m)},(i+1)^{-} ; \boldsymbol{\beta}_{i+1}^{(J)}\right)\right\}}\right\} .
\end{aligned}
$$

The optimal strategy for $\widetilde{V}\left(\widetilde{\mathbf{X}}^{(m)},(i+1)^{-} ; \boldsymbol{\beta}_{i+1}^{(M, J)}\right)$ and $\widetilde{V}\left(\widetilde{\mathbf{X}}^{(m)},(i+1)^{-} ; \boldsymbol{\beta}_{i+1}^{(J)}\right)$ may differ due to the difference between $\boldsymbol{\beta}_{i+1}^{(M, J)}$ and $\boldsymbol{\beta}_{i+1}^{(J)}$. Similar to the argument in previous theorem, the proof has to cover nine cases corresponding to the various combinations of the pair of optimal strategies.

We would like to show that D.2 goes to zero for one typical case: $\gamma_{i+1}^{*(J)}=0$ and $\gamma_{i+1}^{*(M, J)}=G A$. One can show the other cases in a similar manner. For this case, the first term inside the curl bracket of D.2 can be bounded as follows

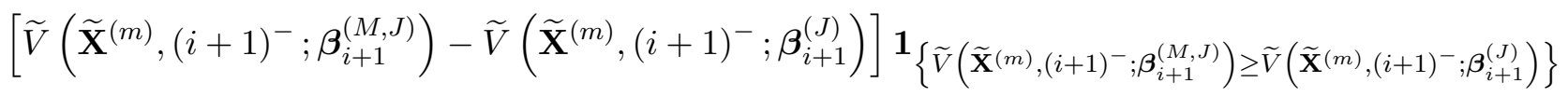

$$
\begin{aligned}
& =\left[G+h_{2}\left(\widetilde{W}_{(i+1)^{-}}, 1 ; G\right) \Psi^{(J)}\left(\widetilde{h}_{1}\left(\widetilde{W}_{(i+1)^{-}}, 1 ; G\right), v\right) \boldsymbol{\beta}_{i+1}^{(M, J)}-h_{2}\left(\widetilde{W}_{(i+1)^{-}}, 1 ; 0\right)\right. \\
& \left.\Psi^{(J)}\left(\widetilde{h}_{1}\left(\widetilde{W}_{(i+1)^{-}}, 1 ; 0\right), v\right) \boldsymbol{\beta}_{i+1}^{(J)}\right] \mathbf{1}_{\left\{\widetilde{V}\left(\widetilde{\mathbf{X}}^{(m)},(i+1)^{-} ; \boldsymbol{\beta}_{i+1}^{(M, J)}\right) \geq \widetilde{V}\left(\widetilde{\mathbf{X}}^{(m)},(i+1)^{-} ; \boldsymbol{\beta}_{i+1}^{(J)}\right)\right\}} \\
& \leq\left[G+h_{2}\left(\widetilde{W}_{(i+1)^{-}}, 1 ; G\right) \Psi^{(J)}\left(\widetilde{h}_{1}\left(\widetilde{W}_{(i+1)^{-}}, 1 ; G\right), v\right) \boldsymbol{\beta}_{i+1}^{(M, J)}-G-h_{2}\left(\widetilde{W}_{(i+1)^{-}}, 1 ; G\right)\right. \\
& \left.\Psi^{(J)}\left(\widetilde{h}_{1}\left(\widetilde{W}_{(i+1)^{-}}, 1 ; G\right), v\right) \boldsymbol{\beta}_{i+1}^{(J)}\right] \mathbf{1}_{\left\{\widetilde{V}\left(\widetilde{\mathbf{X}}^{(m)},(i+1)^{-} ; \boldsymbol{\beta}_{i+1}^{(M, J)}\right) \geq \widetilde{V}\left(\widetilde{\mathbf{X}}^{(m)},(i+1)^{-} ; \boldsymbol{\beta}_{i+1}^{(J)}\right)\right\}}
\end{aligned}
$$

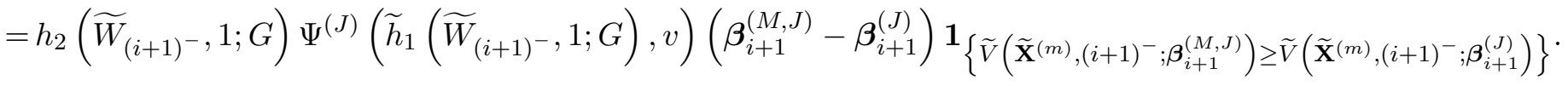


Multiplying $\frac{1}{M} \sum_{m=1}^{M}\left|\Psi^{(J)}\left(\widetilde{\mathbf{X}}_{i}^{(m)}\right)\right|$ and applying the Cauchy-Schwartz inequality, we obtain

$$
\begin{aligned}
& \frac{1}{M} \sum_{m=1}^{M}\left|\Psi^{(J)}\left(\widetilde{\mathbf{X}}_{i}^{(m)}\right)\right|\left[\widetilde{V}\left(\widetilde{\mathbf{X}}^{(m)},(i+1)^{-} ; \boldsymbol{\beta}_{i+1}^{(M, J)}\right)\right. \\
& \left.\quad-\widetilde{V}\left(\widetilde{\mathbf{X}}^{(m)},(i+1)^{-} ; \boldsymbol{\beta}_{i+1}^{(J)}\right)\right] \mathbf{1}_{\left\{\widetilde{V}\left(\widetilde{\mathbf{X}}^{(m)},(i+1)^{-} ; \boldsymbol{\beta}_{i+1}^{(M, J)}\right) \geq \widetilde{V}\left(\widetilde{\mathbf{X}}^{(m)},(i+1)^{-} ; \boldsymbol{\beta}_{i+1}^{(J)}\right)\right\}} \\
& \leq \frac{1}{M} \sum_{m=1}^{M}\left|\Psi^{(J)}\left(\widetilde{\mathbf{X}}_{i}^{(m)}\right)\right|\left|h_{2}\left(\widetilde{W}_{(i+1)^{-}}, 1 ; G\right)\right|\left|\Psi^{(J)}\left(\widetilde{h}_{1}\left(\widetilde{W}_{(i+1)^{-}}, 1 ; G\right), v\right) \|\left(\boldsymbol{\beta}_{i+1}^{(M, J)}-\boldsymbol{\beta}_{i+1}^{(J)}\right)\right| .
\end{aligned}
$$

Similarly, the second term inside the curl bracket of D.2 can be shown to be bounded by

$$
\frac{1}{M} \sum_{m=1}^{M}\left|\Psi^{(J)}\left(\widetilde{\mathbf{X}}_{i}^{(m)}\right)\right|\left|h_{2}\left(\widetilde{W}_{(i+1)^{-}}, 1 ; 0\right)\right|\left|\Psi^{(J)}\left(\widetilde{h}_{1}\left(\widetilde{W}_{(i+1)^{-}}, 1 ; 0\right), v\right)\right|\left|\left(\boldsymbol{\beta}_{i+1}^{(M, J)}-\boldsymbol{\beta}_{i+1}^{(J)}\right)\right|
$$

By the induction assumption, $\boldsymbol{\beta}_{i+1}^{(M, J)}$ converges to $\boldsymbol{\beta}_{i+1}^{(J)}$ almost surely. Hence, for any $\epsilon>0$, lim sup of the summation of (D.3a) and (D.3b) is bounded by

$$
\begin{aligned}
& \limsup _{M \rightarrow \infty} \frac{1}{M} \sum_{m=1}^{M}\left|\Psi^{(J)}\left(\widetilde{\mathbf{X}}_{i}^{(m)}\right)\right|\left[\left|h_{2}\left(\widetilde{W}_{(i+1)^{-}}^{(m)}, 1 ; G\right)\right|\left|\Psi^{(J)}\left(\widetilde{h}_{1}\left(\widetilde{W}_{(i+1)^{-}}^{(m)}, 1 ; G\right), v^{(m)}\right)\right|\right. \\
& \left.\quad+\left|h_{2}\left(\widetilde{W}_{(i+1)^{-}}^{(m)}, 1 ; 0\right)\right|\left|\Psi^{(J)}\left(\widetilde{h}_{1}\left(\widetilde{W}_{(i+1)^{-}}^{(m)}, 1 ; 0\right), v^{(m)}\right)\right|\right] \epsilon \\
& =E\left[| \Psi ^ { ( J ) } ( \widetilde { \mathbf { X } } _ { i } ) | \left[\left|h_{2}\left(\widetilde{W}_{(i+1)^{-}}, 1 ; G\right)\right|\left|\Psi^{(J)}\left(\widetilde{h}_{1}\left(\widetilde{W}_{(i+1)^{-}}, 1 ; G\right), v\right)\right|\right.\right. \\
& \left.\quad+\left|h_{2}\left(\widetilde{W}_{(i+1)^{-}}, 1 ; 0\right)\right|\left|\Psi^{(J)}\left(\widetilde{h}_{1}\left(\widetilde{W}_{(i+1)^{-}}, 1 ; 0\right), v\right)\right|\right] \epsilon .
\end{aligned}
$$

The above equality is due to the law of large numbers. By taking $\epsilon$ to zero, we show that $\boldsymbol{\beta}_{i}^{(M, J)}$ converges to $\boldsymbol{\beta}_{i}^{(J)}$ almost surely.

\section{Appendix E - Proof of Proposition 5}

By the law of large numbers, it suffices to show that as $M \rightarrow \infty$,

$$
\frac{1}{M} \sum_{m=1}^{M}\left[V\left(W^{(m)}, A^{(m)}, v^{(m)}, i^{-} ; \boldsymbol{\beta}_{i}^{(M, J)}\right)-V\left(W^{(m)}, A^{(m)}, v^{(m)}, i^{-} ; \boldsymbol{\beta}_{i}^{(J)}\right)\right] \rightarrow 0, \quad \text { for any } i=1 \text { to } T-1 .
$$

Due to the difference between $\boldsymbol{\beta}_{i}^{(M, J)}$ and $\boldsymbol{\beta}_{i}^{(J)}$ the optimal strategy for $V\left(W^{(m)}, A^{(m)}, v^{(m)}, i^{-} ; \boldsymbol{\beta}_{i}^{(M, J)}\right)$ and $V\left(W^{(m)}, A^{(m)}, v^{(m)}, i^{-} ; \boldsymbol{\beta}_{i}^{(J)}\right)$ may be different. Similar to the argument in Lemma 4, we split the proof to nine cases depending on the different combinations of the pair of optimal strategies.

We would like to show that the result is true for one typical case: $\gamma_{i}^{*(J)}=0$ and $\gamma_{i}^{*(M, J)}=0$. One can show the other cases in a similar manner. For this case, since the optimal strategies are both zero withdrawal, we 
then have

$$
\begin{aligned}
& \left|V\left(W^{(m)}, A^{(m)}, v^{(m)}, i^{-} ; \boldsymbol{\beta}_{i}^{(M, J)}\right)-V\left(W^{(m)}, A^{(m)}, v^{(m)}, i^{-} ; \boldsymbol{\beta}_{i}^{(J)}\right)\right| \\
= & \mid h_{2}\left(W_{i^{-}}^{(m)}, A_{i^{-}}^{(m)} ; 0\right) \Psi^{(J)}\left(\widetilde{h}_{1}\left(W_{i^{-}}^{(m)}, A_{i^{-}}^{(m)} ; 0\right), v^{(m)}\right) \boldsymbol{\beta}_{i}^{(M, J)} \\
& \quad-h_{2}\left(W_{i^{-}}^{(m)}, A_{i^{-}}^{(m)} ; 0\right) \Psi^{(J)}\left(\widetilde{h}_{1}\left(W_{i^{-}}^{(m)}, A_{i^{-}}^{(m)} ; 0\right), v^{(m)}\right) \boldsymbol{\beta}_{i}^{(J)} \mid \\
\leq & \left|h_{2}\left(W_{i^{-}}^{(m)}, A_{i^{-}}^{(m)} ; 0\right) \Psi^{(J)}\left(\widetilde{h}_{1}\left(W_{i^{-}}^{(m)}, A_{i^{-}}^{(m)} ; 0\right), v^{(m)}\right)\right|\left|\boldsymbol{\beta}_{i}^{(M, J)}-\boldsymbol{\beta}_{i}^{(J)}\right| .
\end{aligned}
$$

The above inequality is obtained by the virtue of the Cauchy-Schwarz inequality. By Lemma $4, \boldsymbol{\beta}_{i}^{(M, J)}$ converges to $\boldsymbol{\beta}_{i}^{(J)}$ almost surely. Hence, for any $\epsilon>0$, we have

$$
\begin{aligned}
& \limsup _{M \rightarrow \infty} \frac{1}{M} \sum_{m=1}^{M}\left|V\left(W^{(m)}, A^{(m)}, v^{(m)}, i^{-} ; \boldsymbol{\beta}_{i}^{(M, J)}\right)-V\left(W^{(m)}, A^{(m)}, v^{(m)}, i^{-} ; \boldsymbol{\beta}_{i}^{(J)}\right)\right| \\
\leq & \limsup _{M} \frac{1}{M} \sum_{m=1}^{M}\left|h_{2}\left(W_{i^{-}}^{(m)}, A_{i^{-}}^{(m)} ; 0\right) \Psi^{(J)}\left(\widetilde{h}_{1}\left(W_{i^{-}}^{(m)}, A_{i^{-}}^{(m)} ; 0\right), v^{(m)}\right)\right|\left|\boldsymbol{\beta}_{i}^{(M, J)}-\boldsymbol{\beta}_{i}^{(J)}\right| \\
\leq & \limsup _{M} \frac{1}{M} \sum_{m=1}^{M}\left|h_{2}\left(W_{i^{-}}^{(m)}, A_{i^{-}}^{(m)} ; 0\right) \Psi^{(J)}\left(\widetilde{h}_{1}\left(W_{i^{-}}^{(m)}, A_{i^{-}}^{(m)} ; 0\right), v^{(m)}\right)\right| \epsilon \\
= & E\left[\left|\Psi^{(J)}\left(\widetilde{h}_{1}\left(W_{i^{-}}, A_{i^{-}} ; 0\right), v\right)\right|\right] \epsilon .
\end{aligned}
$$

The above equality is due to the law of large numbers. By taking $\epsilon$ to zero, we obtain the desired result. 


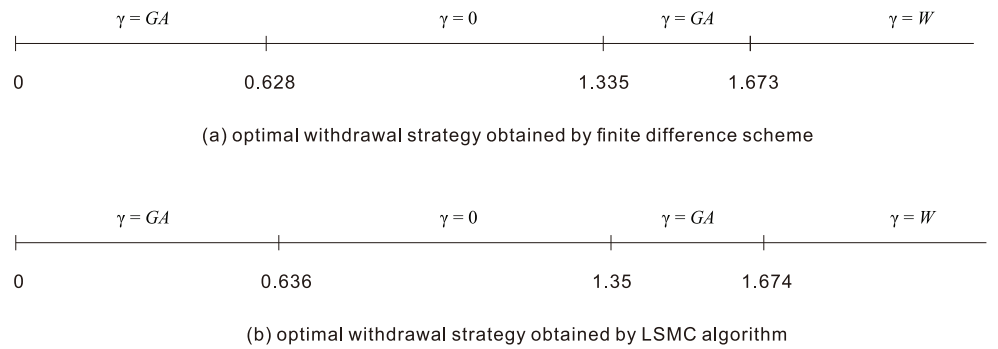

Figure 1: We use (a) finite difference scheme and (b) LSMC algorithm to determine the optimal withdrawal strategies at $t=1$. We observe good agreement of the threshold values of the normalized fund value $W / A$ that separate the domain into different regions of optimal withdrawal strategy: $\gamma=G A, \gamma=0$ and $\gamma=W$.

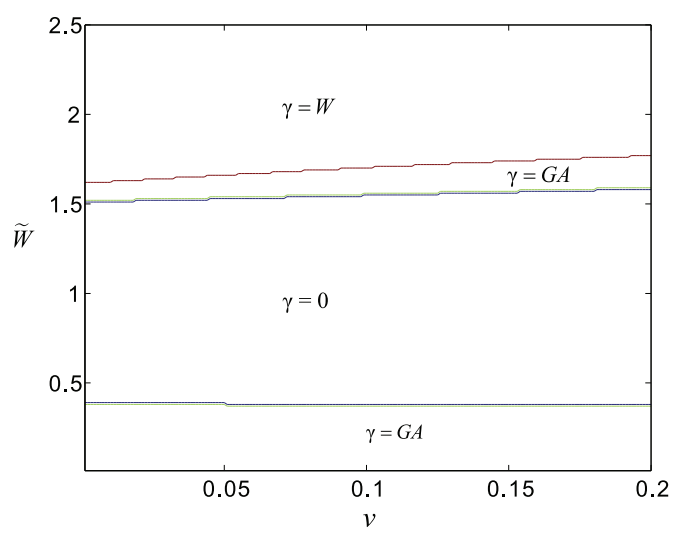

Figure 2: Separation of the optimal withdrawal regions in the $\widetilde{W}-v$ plane at $t=1$ corresponding to the 3 optimal strategies (i) $\gamma=W$, (ii) $\gamma=0$ and (iii) $\gamma=G A$ at $t=1$ under the $3 / 2$-model.
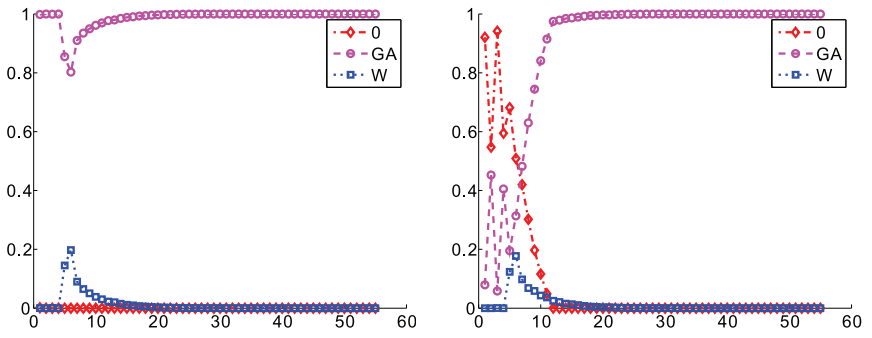

(a) Bonus: $0.04 \%$, Ratchet: Every two years (b) Bonus: $0.07 \%$, Ratchet: Every two years
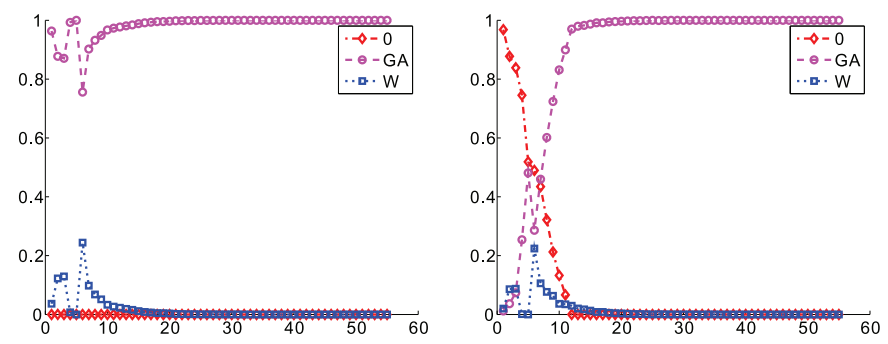

(c) Bonus: $0.04 \%$, Ratchet: Every five years (d) Bonus: $0.07 \%$, Ratchet: Every five years

Figure 3: Percentage of each of the 3 optimal withdrawal strategies (i) $\gamma=0$, (ii) $\gamma=G A$, (iii) $\gamma=W$ over the policy life of the GLWB under the 3/2-model. 

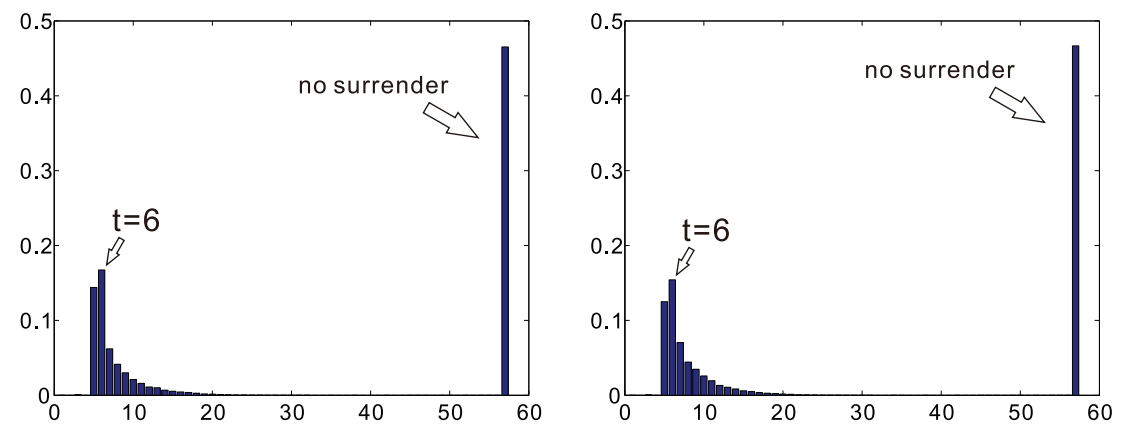

(a) Bonus: $0.04 \%$, Ratchet: Every two years (b) Bonus: $0.07 \%$, Ratchet: Every two years
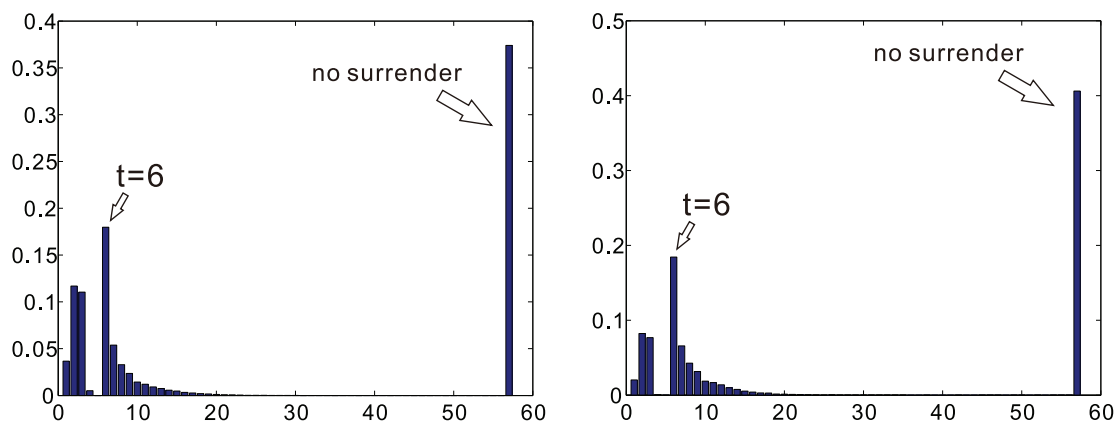

(c) Bonus: $0.04 \%$, Ratchet: Every five years (d) Bonus: $0.07 \%$, Ratchet: Every five years

Figure 4: Impact of the bonus rate and ratchet provision on the distribution of the optimal time for choosing complete surrender over the policy life of the GLWB under the $3 / 2$-model. The penalty charge setting is "Penalty 1".
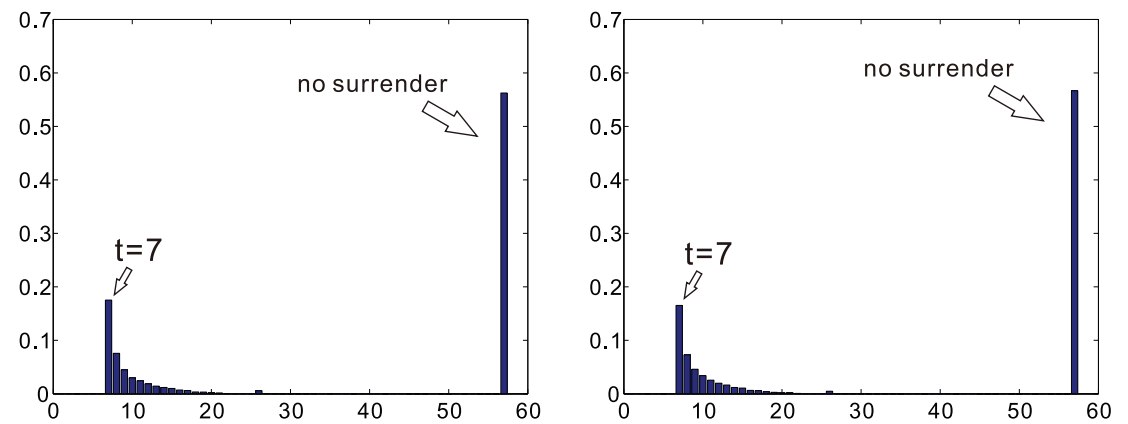

(a) Bonus: $0.04 \%$, Ratchet: Every two years (b) Bonus: $0.07 \%$, Ratchet: Every two years
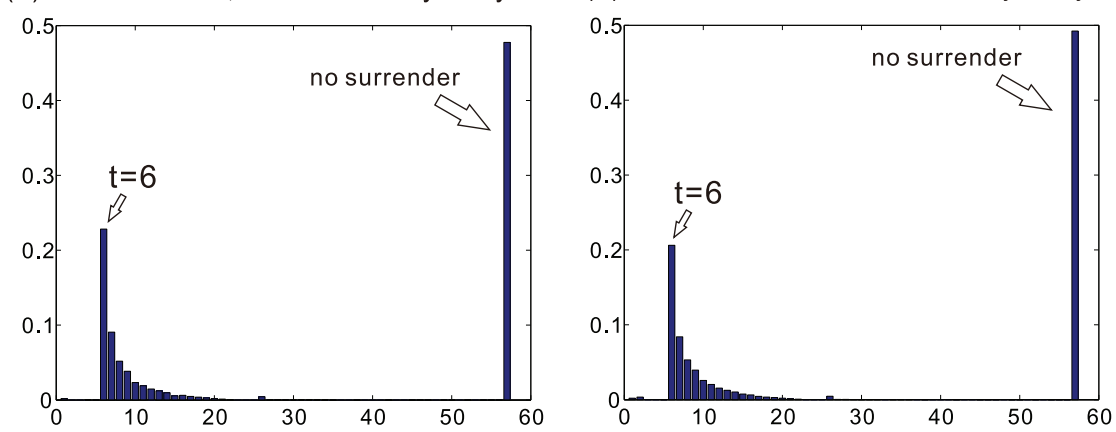

(c) Bonus: $0.04 \%$, Ratchet: Every five years (d) Bonus: $0.07 \%$, Ratchet: Every five years

Figure 5: Impact of the bonus rate and ratchet provision on the distribution of the optimal time for choosing complete surrender over the policy life of the GLWB the under 3/2-model. The penalty charge setting is "Penalty 2". 